\title{
LHCf detector performance during the 2009-2010 LHC run
}

The LHCf Collaboration; O. Adriani ${ }^{1}, 2$, L. Bonechi $^{1}$, M. Bongi ${ }^{1}$, G. Castellini ${ }^{1}$, R.

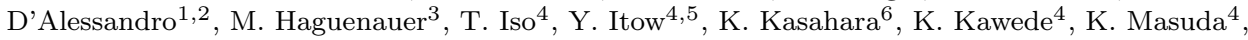

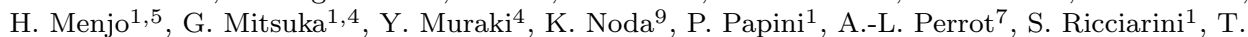

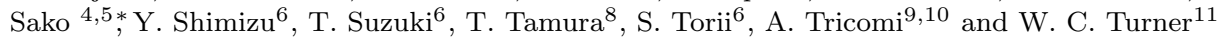

${ }^{1}$ INFN Section of Florence, Italy

${ }^{2}$ University of Florence, Italy

${ }^{3}$ Ecole-Polytechnique, Palaiseau, France

${ }^{4}$ Solar-Terrestrial Environment Laboratory, Nagoya University, Nagoya, Japan

${ }^{5}$ Kobayashi-Maskawa Institute for the Origin of Particles and the Universe, Nagoya University, Nagoya, Japan

${ }^{6}$ RISE, Waseda University, Japan

${ }^{7}$ CERN, Switzerland

${ }^{8}$ Kanagawa University, Japan

${ }^{9}$ INFN Section of Catania, Italy

10 University of Catania, Italy

${ }^{11}$ LBNL, Berkeley, California, USA

Received Day Month Year

Revised Day Month Year

\begin{abstract}
LHCf has successfully completed the operation during the LHC 2009-2010 period and the detectors were removed in July 2010. The event trigger, data analysis and background have been intensively studied in order to derive inclusive single photon and $\pi^{0}$ spectra. In this paper the details of these intensive studies are described.
\end{abstract}

Keywords: LHCf; sampling calorimeter; particle identification.

PACS numbers: $11.25 . \mathrm{Hf}, 123.1 \mathrm{~K}$

\section{Introduction to the LHCf Experiment}

\subsection{Physics and Detectors}

The Large Hadron Collider forward (LHCf) experiment has been designed to measure very forward neutral particles emitted in LHC hadron-hadron collisions. The data are useful for calibration of the hadronic interaction models used in ultra high energy cosmic-ray physics ${ }^{1}$. LHCf has installed two compact detectors in the instrumentation slots of the TANs (Target Neutral Absorbers) located $\pm 140 \mathrm{~m}$ from the interaction point 1 (IP1) as shown in Fig.1. Only neutral particles with pseudorapidity $\eta>8.4$ can reach this location. Charged particles are swept away by the

*corresponding author: sako@stelab.nagoya-u.ac.jp 
inner beam separation dipoles D1 located in the region between IP1 and TANs. Neutral particles with $\eta<8.4$ are lost by hitting the beam pipe before arriving at the LHCf detectors. One detector, named Arm1, was installed on the IP8 side of IP1 and the other, named Arm2, was installed on the IP2 side. Each detector contains two small sampling shower calorimeters (called 'towers' hereafter) with transverse sizes of $20 \mathrm{~mm} \times 20 \mathrm{~mm}$ and $40 \mathrm{~mm} \times 40 \mathrm{~mm}$ for Arm1, $25 \mathrm{~mm} \times 25 \mathrm{~mm}$ and $32 \mathrm{~mm}$ $\times 32 \mathrm{~mm}$ for Arm2 as shown in Fig.2. The longitudinal dimension of the towers was chosen to be 44 radiation lengths or 1.55 hadron interaction lengths of Tungsten interleaved with 16 sampling layers of plastic scintillator. In addition four X-Y pairs of position sensitive detectors, scintillating fiber belts (SciFi) for Arm1 ${ }^{2}$ and silicon strip sensors for Arm $2{ }^{3}$, were inserted in the towers to measure the transverse profile of the showers. The calorimeters have been shown in test beams to have better than $5 \%$ and $200 \mu$ m energy and position resolutions, respectively, for photons with energy greater than $100 \mathrm{GeV} 4{ }^{5}{ }^{6}$. Taking account of electrical noise at the LHC site, the energy resolution is about $8 \% / \sqrt{E / 100 G e V}+1 \%$.

Each LHCf detector is packaged in an aluminum frame with dimensions $92 \mathrm{~mm}(\mathrm{~W}) \times 280 \mathrm{~mm}(\mathrm{~L}) \times 620 \mathrm{~mm}(\mathrm{H})$ and supported in a TAN instrumentation slot by a manipulator. A manipulator is fixed on the top surface of a TAN and allows the LHCf detector to be moved vertically by a $120 \mathrm{~mm}$ stroke. During normal operation with zero degree beam crossing angle, the LHCf detectors were placed $20 \mathrm{~mm}$ above their lowest position so that zero degree collision angle was centered on the small towers. Depending on the beam crossing angle at IP1, where the colliding beams are directed downward, the LHCf detectors are moved downward to increase the rapidity coverage. When LHC operations were not producing collisions, or more precisely when the 'stable beams' condition was not declared, the LHCf detectors were placed at their highest position ('garage position') to minimize radiation damage.

The LHCf Front Counters (FCs) were installed in front of each main detector. The FCs are simple thin plastic scintillators. They were used to monitor the beambeam collision rates with a higher detection efficiency than the LHCf towers. For each FC, two pairs of thin plastic scintillators $(40 \mathrm{~mm} \times 80 \mathrm{~mm})$ were aligned in the vertical and horizontal directions to compose a double layer counter as shown in Fig.3. A $0.5 \mathrm{~mm}$ thick copper plate was inserted between the two layers to absorb low energy electrons emitted by the radioactive isotopes. A coincidence signal of the two layers was used for beam monitoring discussed in Sec.2.3. The positions of the FCs were fixed to the TAN and not moved by the manipulators.

\subsection{LHCf Operation during 2009-2010}

LHCf collected data at LHC from 6 December 2009 to 19 July 2010. By that time an integrated luminosity of about $350 \mathrm{nb}^{-1}$ of proton-proton collisions had been delivered at IP1. This provided a sufficient number of events to achieve the physics program of LHCf. Because of the severe radiation environment and development of 
high energy showers in the detectors, the plastic scintillators were expected to suffer from radiation and the light yield was expected to degrade by about $10 \%$ by the time an integrated luminosity of $350 \mathrm{nb}^{-1}$ was reached. To avoid further radiation damage and multi-hit pileup events at increasing luminosity, the LHCf detectors were removed from the LHC tunnel during a technical stop on 20 July 2010.

Most of the LHCf data were taken for $7 \mathrm{TeV}$ collisions in 2010. A small amount of data for $900 \mathrm{GeV}$ collisions in 2009 and $0.3 \mathrm{nb}^{-1}$ in 2010 (2,3 and 27 May 2010) were also collected. Data were taken only when the 'stable beams' condition was declared by LHC operations. Because it took 10 min to move the LHCf detectors from their garage positions to their data taking positions and then back out when a programmed beam dump was declared, the durations of the LHCf physics operation periods were about 20 min shorter than the declared duration of each 'stable beams' period.

Until 5 June 2010, LHC provided stable beams with zero degree beam-beam crossing angle and then from 25 June 2010 turned to setting a downward crossing angle of $100 \mu \mathrm{rad}$ for each beam (200 $\mu \mathrm{rad}$ total beam-beam crossing angle). To continue covering zero degree collision angle with the small towers, the LHCf detectors were positioned $14 \mathrm{~mm}$ below their normal positions for zero degree beambeam crossing angle. Simultaneously with this increase in beam-beam crossing angle, the maximum beam intensity increased from $2 \times 10^{10} \mathrm{ppb}$ (protons per bunch) to $1 \times 10^{11} \mathrm{ppb}$.

In this paper, we review the performance of the LHCf detectors during 20092010 operation of LHC. In Sec.2, the performance of the trigger, the event counting statistics and the determination of luminosity are described. In Sec.3, the perfor-

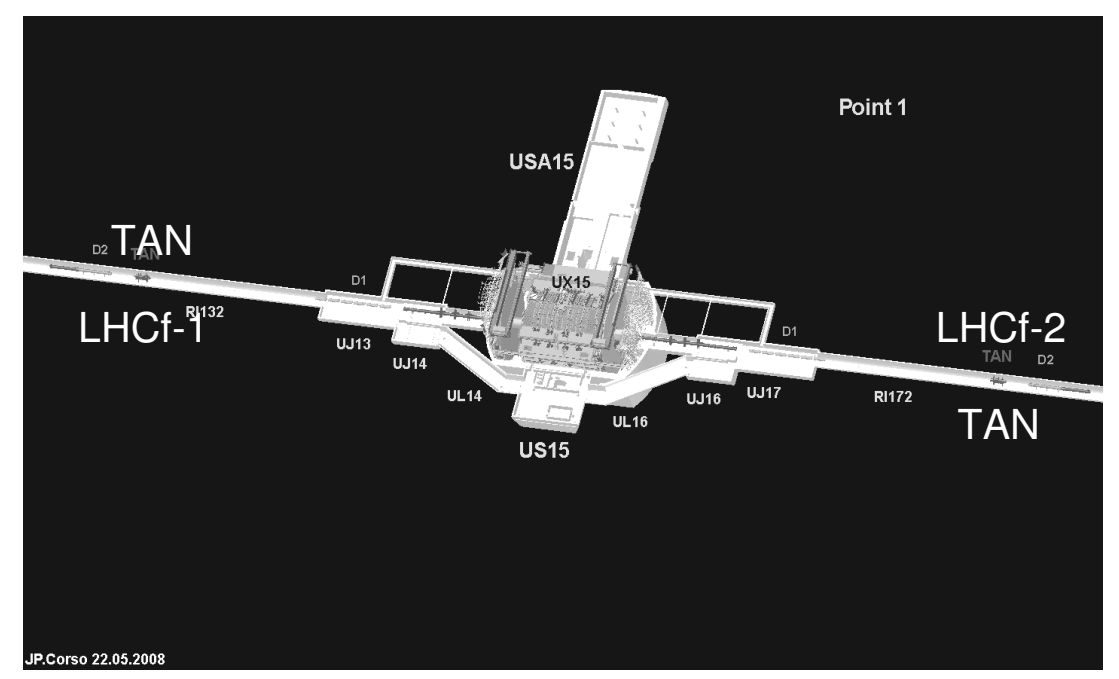

Fig. 1. Location of the LHCf detectors at IP1. The LHCf detectors were installed in the TAN absorbers located $\pm 140 \mathrm{~m}$ from the ATLAS detector (at the center of the figure). 

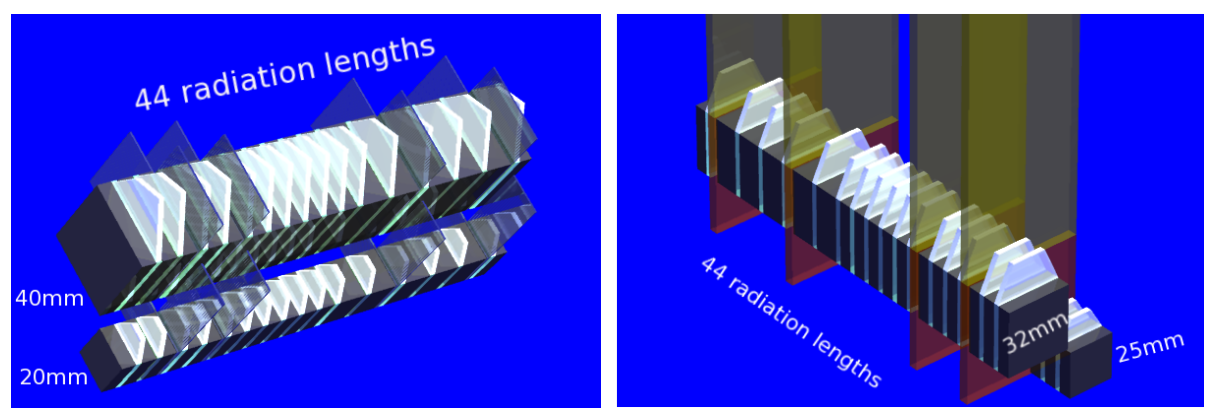

Fig. 2. Schematic views of the LHCf Arm1 (left) and Arm2 (right) detectors.

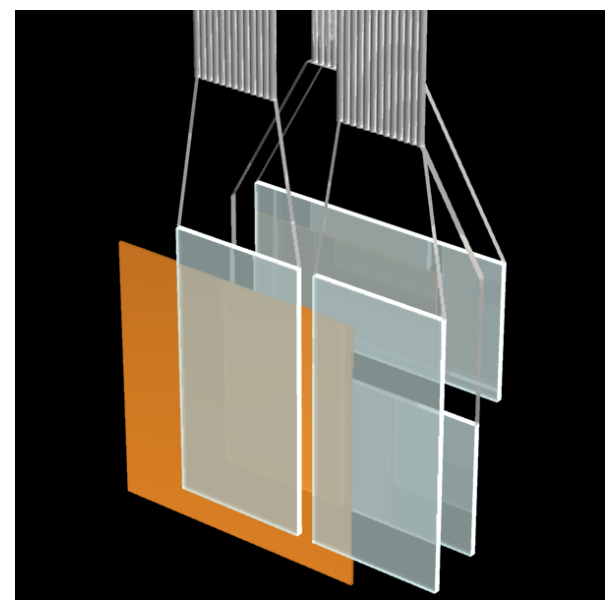

Fig. 3. Schematic view of the LHCf Front Counter.

mance of photon event analysis is described. Performance of neutron event analysis will be described in a later paper. The components of photon performance that are discussed in Sec.3 are: energy and position determinations, particle identification, single/multi-hit identification, beam center determination and $\pi^{0}$ identification. Sec. 4 and Sec.5 describe the effect of beam-gas background and long term stability of the data. Finally a summary is given in Sec.6.

\section{Trigger Performance}

\subsection{LHCf Trigger}

LHCf generated trigger events at three levels and recorded data when an event passed the third level. The trigger and recording of data were in principle independent between Arm1 and Arm2, but a common trigger was also available as one of the options. Independently from the common trigger, trigger flags (true or false infor- 
mation) of the opposite detector were always recorded by both systems to identify common events in offline analysis.

The first level trigger (L1T) was issued according to the BPTX signal which is generated when an incoming beam bunch passes $175 \mathrm{~m}$ upstream the interaction point. The combination of the two BPTX signals corresponding to the two beams was selectable. By default, any one of the BPTXs was used in order to allow L1T triggers to be generated for events without a crossing bunch in the opposite beam. Once the L1T trigger was issued, the gate signal of the charge ADC for the calorimeters was generated. If a third level trigger (L3T) was not issued within $1.5 \mu \mathrm{sec}$, the $\mathrm{ADC}$ was cleared using a fast clear signal and the system waits for the next L1T trigger.

A shower trigger was issued when a high energy shower was identified in any of the calorimeters. The threshold of each sampling layer was set near $17 \mathrm{MeV}$ and $60 \mathrm{MeV}$ of deposited energy for the $900 \mathrm{GeV}$ and $7 \mathrm{TeV}$ collisions, respectively. When the signals in any successive three layers exceeded this level, a shower trigger was generated without regard to whether or not the data acquisition system was ready to record data. A second level trigger (L2T) was issued when any trigger was identified to occur during the period with the data acquisition system was ready to record data. A L2T trigger issued by a shower trigger is called L2TA. L2T triggers issued by a shower trigger in the opposite arm (L2TB), a hit signal from the Front Counters (L2TC) and a delayed L1T signal $\left(\mathrm{L}_{2} \mathrm{~T}_{L 1 T}\right)$ are also available. Because a L1T trigger was issued every time a bunch passed the IP regardless of the existence of interaction, a $\mathrm{L}_{2} \mathrm{~T}_{L 1 T}$ trigger provides an unbiased data sample. The last and highest level trigger (L3T) was issued according to a selectable combination of the various $\mathrm{L} 2 \mathrm{~T}$ triggers and all data with an $\mathrm{L} 3 \mathrm{~T}$ trigger were recorded.

After 25 June 2010 LHCf operated with a special trigger condition owing to the higher luminosity than before and the increased data acquisition dead time. Under this condition, the rate of single shower triggers L2TA was scaled down by a factor $1 / 4$ to $1 / 32$. However all the events with showers in two towers were accepted in order to enhance the trigger efficiency for the $\pi^{0} \rightarrow \gamma \gamma$ and $\eta \rightarrow \gamma \gamma$ events.

For each event the status of the various trigger levels, beam counters and flags were recorded in addition to the Arm1 and Arm2 shower data. The ATLAS level 1 accept signal (ATLAS L1A) was recorded for each event together with the accumulated number of ATLAS L1A signals reset by the common Event Counter Reset signal. Also the timing of each event with the precision of the $40 \mathrm{MHz}$ clock was recorded. The identification of the common LHCf and ATLAS events was confirmed in dry runs performed in 2008 before the operation of LHC.

\subsection{Efficiency and Statistics}

The trigger efficiencies for photons were determined by means of Monte Carlo (MC) simulations and minimum bias trigger data. In the MC simulations the L2TA trigger was simulated taking into consideration the gain of each PMT and the calibration 
Table 1. Summary of the event statistics

\begin{tabular}{cccccc}
\hline & Event & & & \multicolumn{2}{c}{ Number of events } \\
\cline { 4 - 5 }$\sqrt{s}$ & category & Period & Duration & Arm1 & Arm2 \\
\hline $900 \mathrm{GeV}$ & Shower & 6-15 Dec 2009 & $27^{h} 47^{m}$ & 2,840 & 3,731 \\
& & 2, 3, 27 May 2010 & $14^{h} 34^{m}$ & 44,389 & 62,916 \\
\hline $7 \mathrm{TeV}$ & Shower & 30 Mar - 5 Jun 2010 & $223^{h} 40^{m}$ & $59,211,722$ & $54,304,077$ \\
& & 25 Jun - 19 Jul 2010 & $148^{h} 54^{m}$ & $113,051,533$ & $106,283,229$ \\
\cline { 2 - 5 } & $\pi^{0}$ & 14 May - 5 Jun 2010 & & $108 \mathrm{k}$ & $183 \mathrm{k}$ \\
& & 25 Jun - 19 Jul 2010 & & $1,608 \mathrm{k}$ & $1,685 \mathrm{k}$ \\
\hline
\end{tabular}

of pulse height to deposited energy. The trigger efficiencies for photons uniformly incident on the small tower as a function of energy are given in Fig.4 for the low gain operation case which corresponds to $7 \mathrm{TeV}$ collisions. For estimating trigger efficiency from the experimental data, events recorded by the L2TB trigger are selected and the fraction of those events that have both L2TA and L2TB triggers as a function of the reconstructed energy are regarded as the trigger efficiency. Almost $100 \%$ efficiency is achieved above $100 \mathrm{GeV}$ and there is a good agreement between the data and MC simulations. For high gain operation during $900 \mathrm{GeV}$ collisions, $100 \%$ efficiency above $30 \mathrm{GeV}$ is based on only the MC simulation.

Using the L2TA trigger, the mean data acquisition efficiencies were $85.7 \%$ and $67.0 \%$ for the Arm1 and Arm2 detectors, respectively, during $7 \mathrm{TeV}$ collisions with a luminosity of $6 \times 10^{28} \mathrm{~cm}^{-2} \mathrm{~s}^{-1}$. During the $900 \mathrm{GeV}$ collisions with $\mathrm{L}=3$ $12 \times 10^{27} \mathrm{~cm}^{-2} \mathrm{~s}^{-1}$, they were $99.2 \%$ and $98.0 \%$. Here the data acquisition efficiency is defined as $\mathrm{N}_{L 2 T A} / \mathrm{N}_{\text {shower }}$ where $\mathrm{N}_{\text {shower }}$ and $\mathrm{N}_{L 2 T A}$ are the numbers of counts in the shower and L2TA triggers, respectively.

The statistics of the number of recorded events with different collision energies $(900 \mathrm{GeV}$ and $7 \mathrm{TeV}$ ), different operation periods and different event categories (single shower or $\pi^{0}$ candidate) are summarized in Tab.1 separately for Arm1 and Arm2. As described in Sec.3.5, $\pi^{0}$ events have been identified for the $7 \mathrm{TeV}$ data. The number of the candidate $\pi^{0}$ events has been estimated for the data taken after 14 May 2010 when the fine tuning of the $7 \mathrm{TeV}$ data acquisition system (latency mainly) had been completed. These data were taken under the nominal low gain setting. The time history of the event statistics during the $7 \mathrm{TeV}$ operation is shown in Fig.5.

\subsection{Luminosity determination}

The luminosity during LHCf operation was monitored by the Front Counters (FCs) installed in front (IP side) of each of the LHCf main detectors. The normalizations of the FC counting rates to the absolute luminosity were determined by the van der Meer scans carried out on 26 Apr and 9 May, 2010 during $7 \mathrm{TeV}$ collisions. Fig.6 shows an example of the vertical van der Meer scan that took place on 26 Apr. The red and blue curves indicate the counting rates of the Arm1 and Arm2 FCs, respectively, as functions of the beam displacement. Each profile is well fit by two Gaussians and a first order polynomial as shown by the solid curve and residual 

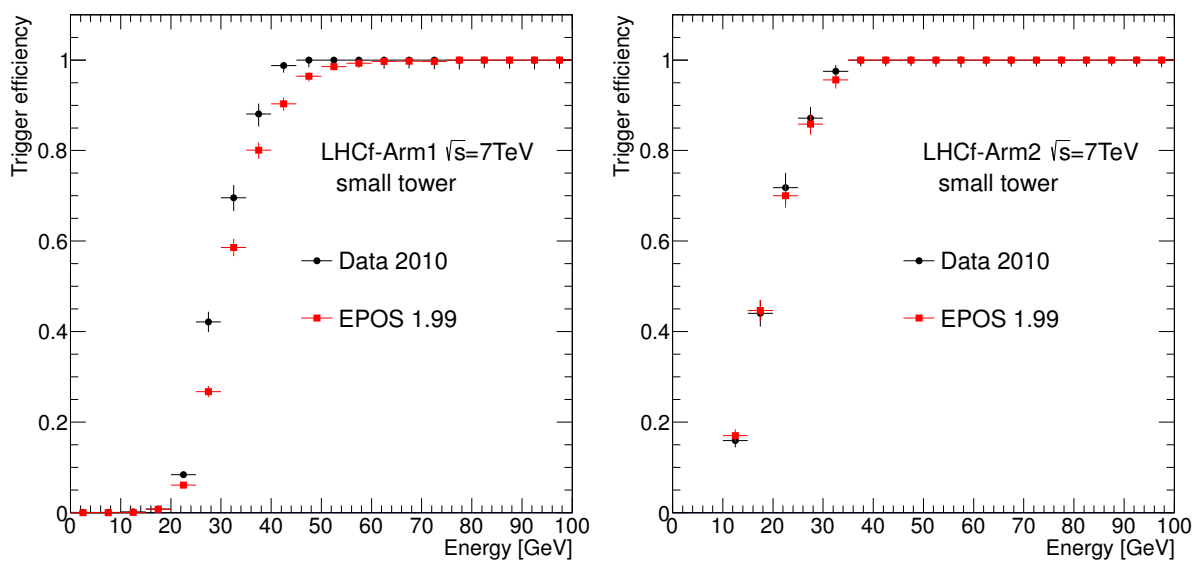

Fig. 4. Trigger efficiencies for photons at low gain setting. The results for the Arm1 (left) and Arm2 (right) small towers are presented. The results for data driven estimation (black) and MC (red) are shown.

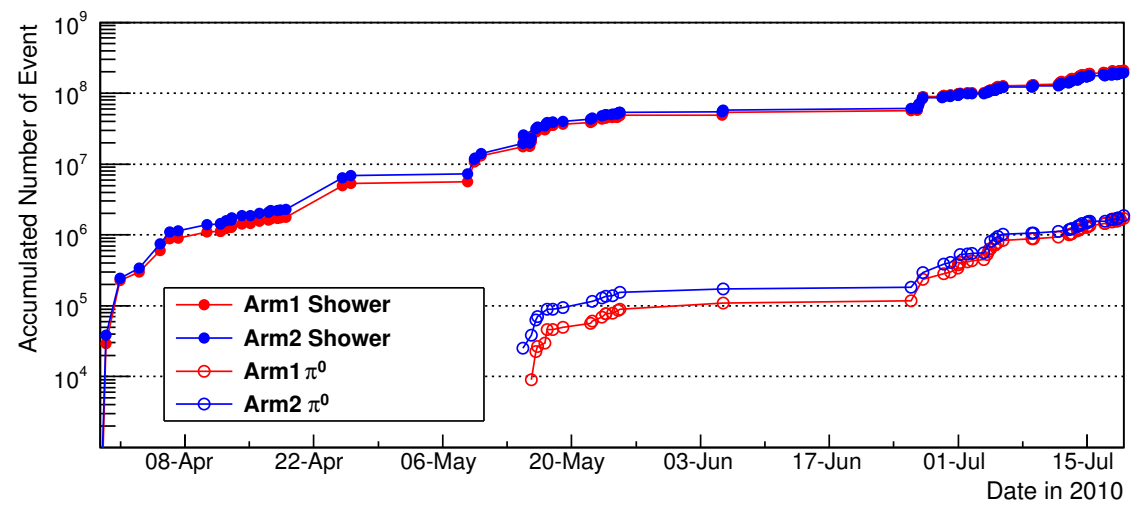

Fig. 5. Accumulated number of events during $7 \mathrm{TeV}$ data taking in 2010. Red and blue plots show the results of Arm1 and Arm2, respectively, where filled and open circles indicate the number of single shower and $\pi^{0}$ candidates, respectively.

in the bottom panel. Here one of the Gaussians dominates the overall structure but the other Gaussian and the polynomial function improve the fit in the tail of the distribution. The absolute luminosity at the peak of the FC counting rate can be derived from the beam width defined by the width of the van der Meer profile, the measured beam intensity and some other beam parameters. Conversion factors from $\mathrm{FC}$ counting rate to luminosity are derived from the absolute luminosity measured by the van der Meer scans and the FC counting rates at the peak. From the difference between the scans on 26 Apr and 9 May 2010, the results from FCs 


\section{LHCf Collaboration}

at Arm1 and Arm2 and the uncertainty in the beam intensity determination, the FCs can provide luminosity with a $\pm 5 \%$ uncertainty. More details of the FCs and luminosity determination are described in a previous report ${ }^{7}$.
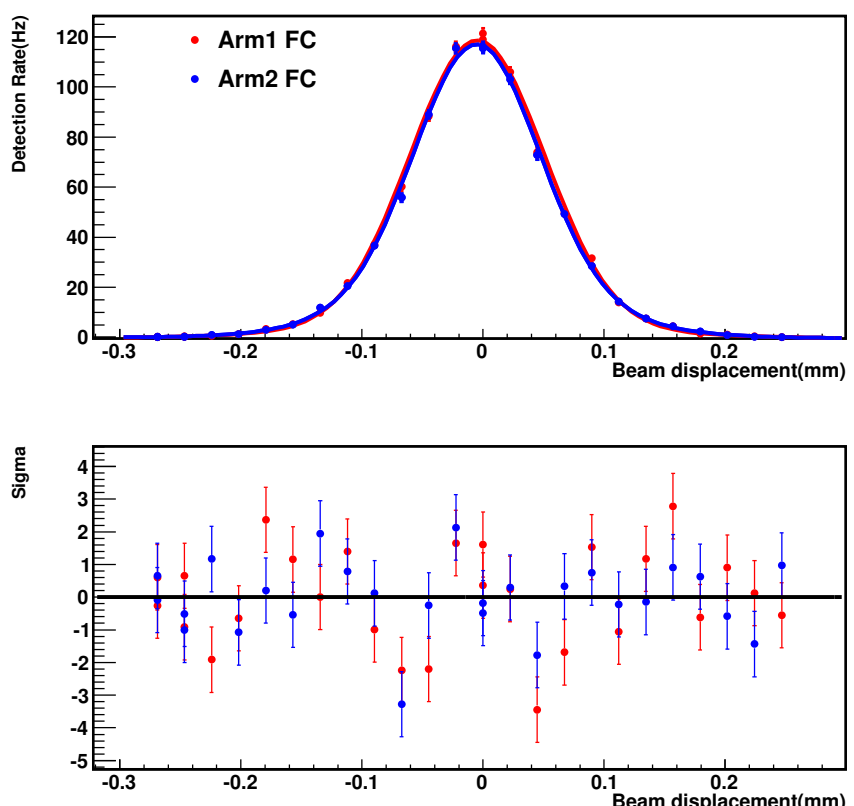

Fig. 6. An example of a van der Meer scan. Red and blue dots of the top plot indicate the counting rates of the Arm1 and Arm2 FCs, respectively, as functions of beam displacement. The bottom plot shows the residual of fits to the data divided by the error of each bin.

\section{Photon Data Analysis}

LHCf data analysis has so far concentrated on photon events taken during low luminosity operation 8910 . The data analysis described in this paper is mainly limited to photon events with luminosity below $10^{29} \mathrm{~cm}^{-2} \mathrm{~s}^{-1}$ during $7 \mathrm{TeV}$ collisions. Some results for $900 \mathrm{GeV}$ collisions are also presented based on the data taken in May 2010. Analysis of $900 \mathrm{GeV}$ data taken in 2009 gave us only small statistics under relatively higher background than in May 2010 and is neglected in this paper. An example of an LHCf event recorded by the Arm2 detector is shown in Fig.7. Two showers, one each hitting the small and large towers, are identified. In the top panels, the longitudinal development of the showers is shown. One layer corresponds to two radiation lengths up to the $10^{\text {th }}$ layer and then four radiation lengths from the $11^{\text {th }}$ layer onward. The middle and the bottom panels indicate the lateral spread of the showers measured by the silicon strip detectors projected in the two orthogonal 
directions, $\mathrm{X}$ and $\mathrm{Y}$. Different colors show the profiles at four different depths $(6,12$, 30 and 42 radiation lengths). Based on the longitudinal and lateral profiles, energy, position and type of the incident particles are reconstructed.
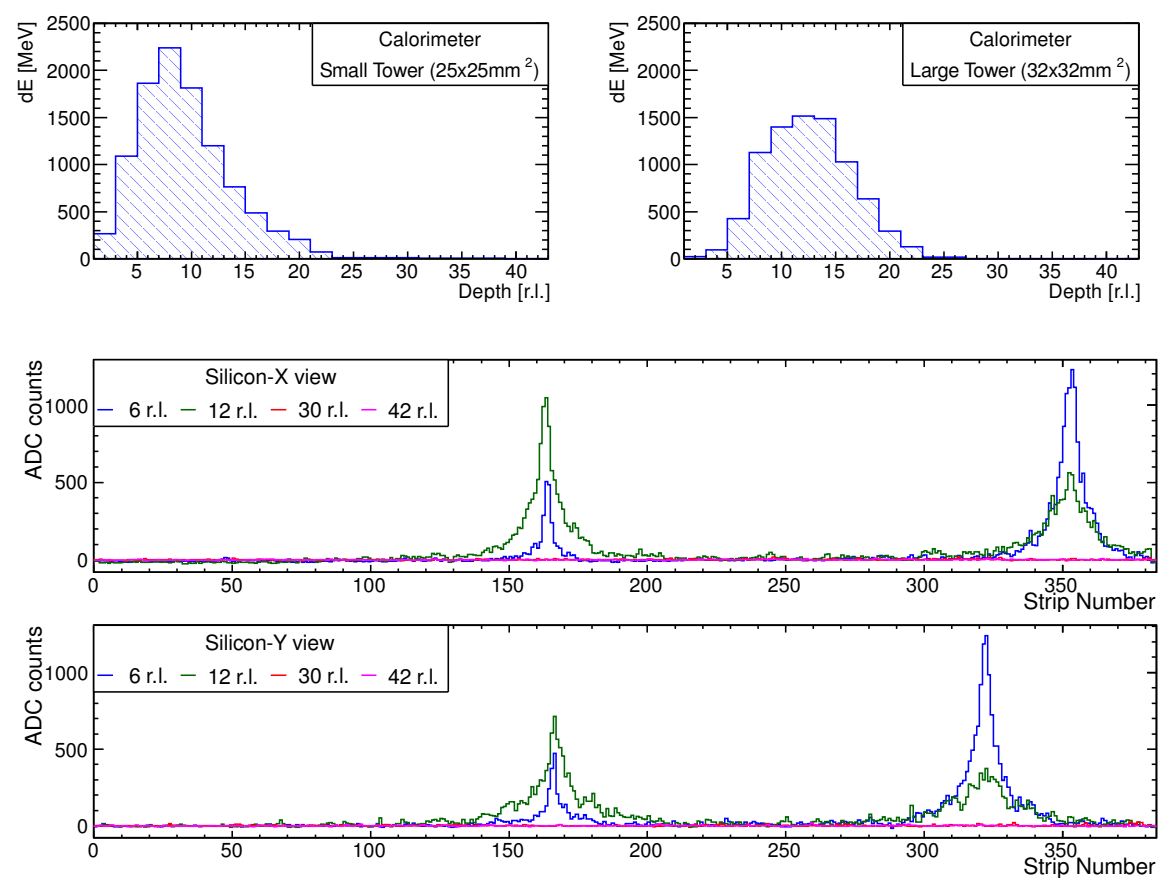

Fig. 7. An example of an LHCf event measured by the Arm2 detector, in this case an event with a single primary photon in each tower. The top plots are the longitudinal shower developments in the two towers. The middle and bottom plots are the lateral distributions of the shower particles measured by the silicon strip sensors. Positions of the two showers are clearly identified. Different colors show the data from four different depths. The middle panel is for the $\mathrm{X}$ projection and the bottom for Y.

\subsection{Energy and Position Determination}

Peak positions in the SciFi and Silicon strip lateral distributions are searched for first by using the program TSpectrum ${ }^{11}$ class in ROOT ${ }^{12}$. Based on the list of peak positions given by TSpectrum, the lateral data are fit with Lorentzian functions to obtain more precise estimates of position, peak height and width. Fig.8 shows examples of fitting in case of multiple showers in the Arm1 (two showers in the large tower) and Arm2 (two showers in the small tower and a single shower in the large tower; A single layer of the silicon strip sensor covers both the small and large towers) detectors. The position resolutions have been studied using SPS electron beam data below $200 \mathrm{GeV}$ and $\mathrm{MC}$ simulations ${ }^{2}{ }^{3}$. The distributions of 
the reconstructed shower incident position for photon-like events during the LHC $7 \mathrm{TeV}$ operation are shown in Fig.9. The coordinates are as seen from the IP to the detectors. The $\mathrm{Y}$ axes are along the vertical direction and the $\mathrm{X}$ axes horizontally direct to the outside and inside of the LHC ring in the plots of Arm1 and Arm2, respectively. Clear edges are found in the vertical distributions of photon-like events in the large towers. These edges correspond to the shadow of the beam pipe projected onto the detector plane. A detailed study of the incident photon position determination for single-hit, multi-hit events is discussed in Sec.3.3.
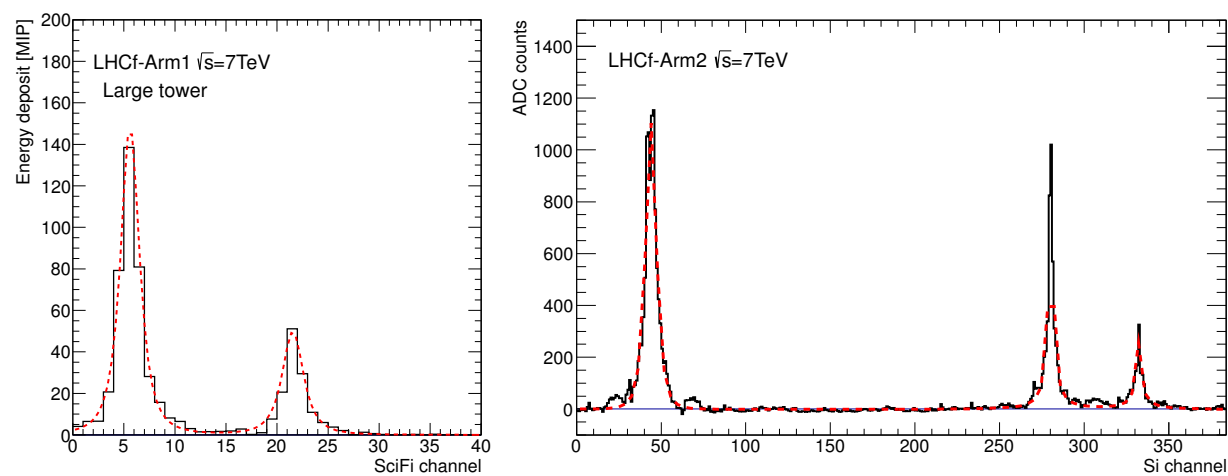

Fig. 8. An example of the lateral distributions for a multi hit event and fitting with Lorentzian functions for Arm1 (left) and Arm2(right).

Once the incident photon position is determined, the position dependent light collection efficiency and shower leakage corrections are applied. Summation of the energies deposited in the $2^{\text {nd }}$ to the $13^{\text {th }}$ layers are converted to the primary photon energy based on MC simulations. The performance of the detector for electromagnetic showers was tested below $200 \mathrm{GeV}$ at the SPS fixed target electron beam ${ }^{6}$. Note the energy assigned here is valid only for an electromagnetic primary. The verification of primary photon energy determination during LHC operation is discussed in Sec.3.5 which deals with the reconstruction of $\pi^{0}$ events.

\subsection{Particle Identification (PID)}

Particles detected by the LHCf detectors are predominantly photons and neutrons (and $\Lambda$ in the low energy range). At the detector level electromagnetic or hadronic identification is performed using a parameter $\mathrm{L}_{90 \%}$. As illustrated in Fig.10, $\mathrm{L}_{90 \%}$ is the longitudinal distance in radiation length measured from the entrance to a tower to the position where $90 \%$ of the total shower energy recorded in the towers has been deposited.

Fig.11 shows the distribution of $\mathrm{L}_{90 \%}$ for the Arm1 small tower for events with reconstructed energy between $50 \mathrm{GeV}$ and $100 \mathrm{GeV}$ (900 GeV collision data) and 

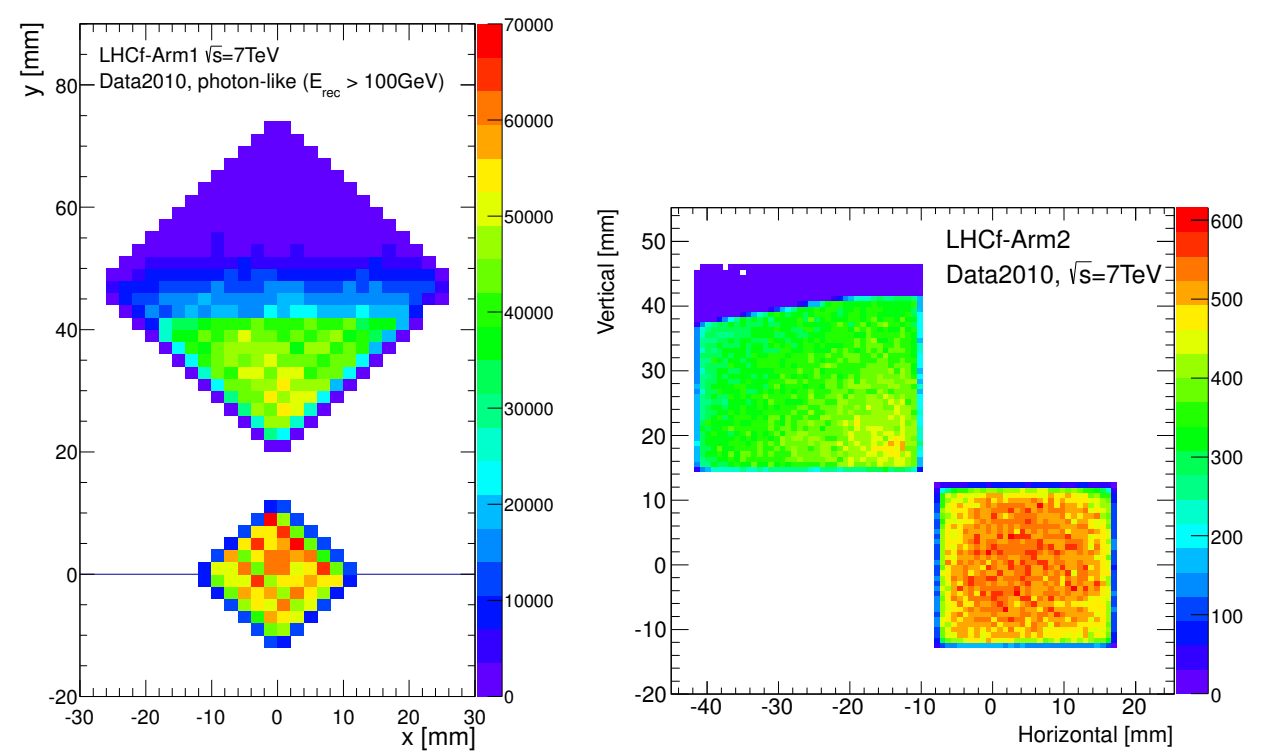

Fig. 9. Distribution of the incident position of the photon-like events observed by Arm1 (left) and Arm2 (right) detectors. Color scales indicate the number of events recorded in each cell. The definition of the coordinate system is given in the text.

between $500 \mathrm{GeV}$ and $1 \mathrm{TeV}$ ( $7 \mathrm{TeV}$ collision data). Two distinct peaks are observed corresponding to photon and neutron events. Photon-like events can be selected by applying a threshold in $\mathrm{L}_{90 \%}$ at around 20 radiation length. Some fine structures such as a small peak at 25 radiation length are produced due to the discrete sampling of the calorimeters and appear both in the experimental data and MC simulations. The $\mathrm{L}_{90 \%}$ distributions for pure photon and neutron samples are generated by MC simulation using the collision product generator QGSJET II-0 $0{ }^{13}$ as shown in Fig.11 (scaled to data as explained below). They are called 'templates' hereafter. The choice of a specific interaction model is not essential here. In the event selection, an energy dependent threshold in $\mathrm{L}_{90 \%}$ was set to keep the photon detection efficiency $\epsilon_{P I D} \sim 90 \%$ over the entire energy range based on the photon template. The purity of the selected photon events is determined by scaling the template functions to the observed $\mathrm{L}_{90 \%}$ distribution. The scaling factors are independently determined for photon and hadron templates. The purity, $\mathrm{P}$ of photon events, is defined as $\mathrm{P}=$ $\mathrm{N}_{\text {phot }} /\left(\mathrm{N}_{\text {phot }}+\mathrm{N}_{\text {had }}\right)$ in each energy bin. Here $\mathrm{N}_{\text {phot }}$ and $\mathrm{N}_{\text {had }}$ are the numbers of photon and neutron events below the threshold of $\mathrm{L}_{90 \%}$ in the scaled templates .

Fig.12 shows the $\mathrm{L}_{90 \%}$ distributions of the Arm1 small tower in different energy ranges. The separation of the photon and neutron distributions decreases as the energy increases. Because the length of the tower is only 1.55 hadron interaction lengths, a large energy deposition by a hadronic shower requires an early shower development in the tower and consequently occurs for a smaller $\mathrm{L}_{90 \%}$. It is also 
remarked that the primary energy assigned here is equivalent to the electromagnetic shower energy discussed in Sec.3.1. Because of the large leakage of the hadronic shower particles from the ends of the calorimeters, there is essentially a very small number of neutron events in the $2 \mathrm{TeV}<\mathrm{E}_{\text {recon }}<3.5 \mathrm{TeV}$ bin. On average a $3 \mathrm{TeV}$ neutron primary shower produces an electromagnetic equivalent energy of $1 \mathrm{TeV}$. The efficiency and purity of the photon samples as functions of the energy are shown in Fig.13. In the spectral analysis non-biased photon energy spectra are obtained by multiplying each energy bin content by $\mathrm{P} \times \epsilon_{P I D}^{-1}$.
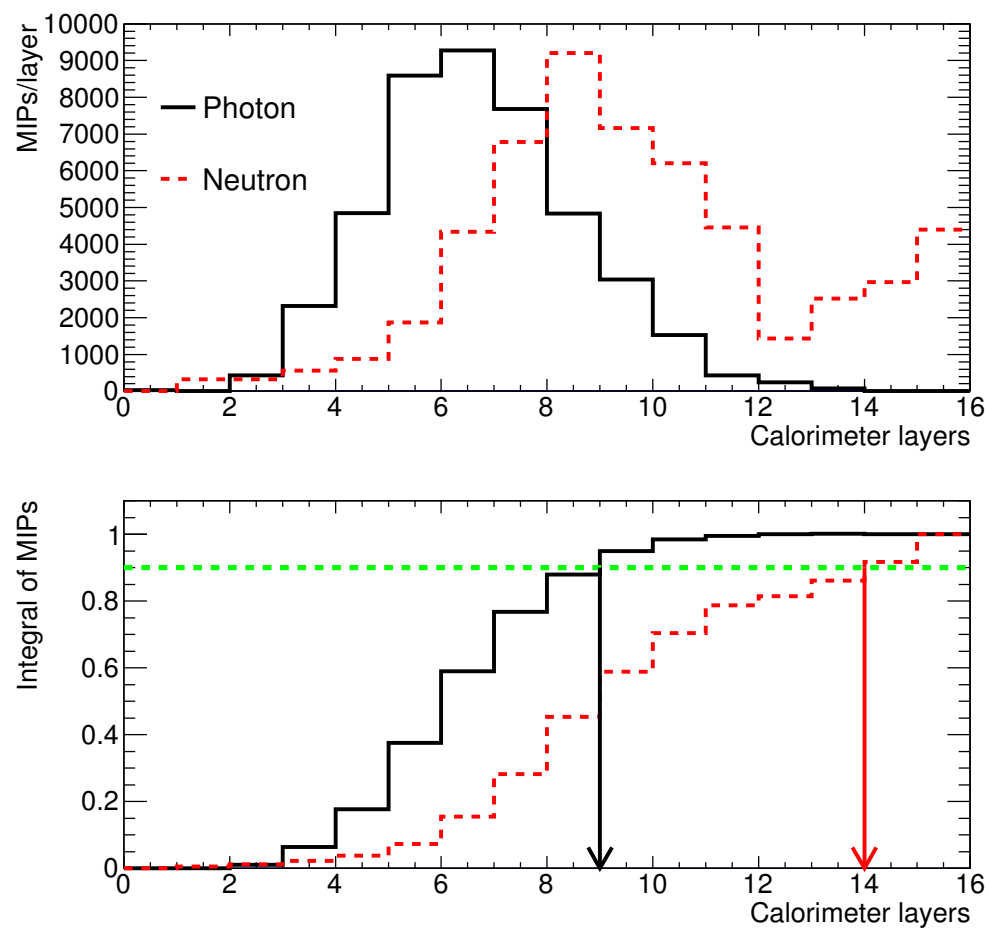

Fig. 10. Definition of $\mathrm{L}_{90 \%}$. The top plot shows typical longitudinal developments of electromagnetic (black solid line) and hadronic (red dashed line) showers in a LHCf tower. The bottom plot shows the accumulated energy normalized to 1.0 at the end of the calorimeters. The depth in the tower (shown by arrows) where the accumulation reaches 0.9 is defined as $\mathrm{L}_{90 \%}$.

In Fig.11 apparent mismatches between the data and the MC templates are found. These differences give rise to different estimates of photon efficiency and purity and therefore contribute to systematic errors in the final spectral analysis. To estimate the systematic errors, the template distributions were artificially modified and another set of values for efficiency and purity were estimated. The difference in the spectra from the two methods (original (A) and modified (B)) is regarded as a systematic uncertainty of the PID. The artificial modification of the template 

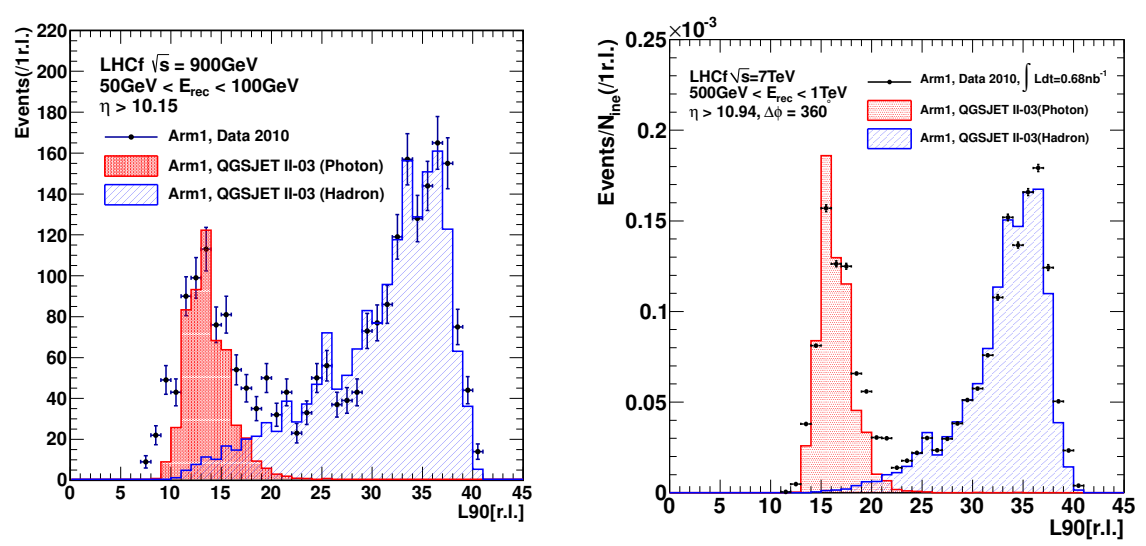

Fig. 11. Observed (crosses) and template (histograms) $\mathrm{L}_{90 \%}$ distributions in the $50-100 \mathrm{GeV}$ (left) and $500-1000 \mathrm{GeV}$ (right) energy ranges for the $900 \mathrm{GeV}$ and $7 \mathrm{TeV}$ collision data, respectively. Templates are scaled to fit the observed data with independent scaling factors for photons and hadrons.

distributions allows for a shift of the peak position and a broadening until obtaining the best match with the data. Finally a maximum 0.7 radiation lengths shift and $20 \%$ broadening for the $7 \mathrm{TeV}$ data and 1 radiation length shift and $30 \%$ broadening for the $900 \mathrm{GeV}$ data were required to obtain the best matches between the data and the MC. The correction factors, $\mathrm{P} \times \epsilon_{P I D}^{-1}$, obtained by two methods $\mathrm{A}$ and $\mathrm{B}$ described above are shown in Fig.14. The differences of correction factors are $\pm 5 \%$ below $1700 \mathrm{GeV}$ and $\pm 20 \%$ above $1700 \mathrm{GeV}$ for the Arm 1 small tower, for example. These differences are included as systematic errors in the photon spectra.

\subsection{Multi-hit Identification}

In the LHCf shower analysis, the events with more than one shower in a single tower are eliminated to extract the correct primary particle energy. The efficiency of single photon identification has been studied based with MC simulations. Lateral distribution fitting described in Sec.3.1 is performed assuming that multiple showers exist. If a second peak in any of the X-Y layers of the first and second position sensitive layers is clearly separated from the first peak and it has a sufficient peak height and reasonable lateral shape, the event is identified as a multi-hit event and eliminated in the analysis. When the conditions above are not satisfied, the event is classified as a single-hit event and used in the analysis. With typical criteria for identifying multi-hit events, for example more than $1 \mathrm{~mm}$ peak separation and more than $5 \%$ of the energy in the second peak, $>98 \%$ of the simulated single-hit events are correctly identified. The detailed criteria for identifying multi-hit events are optimized separately for each Arm. Fig.15 shows the single-hit identification efficiencies of the Arm1 and Arm2 small towers as a function of the photon energy. 

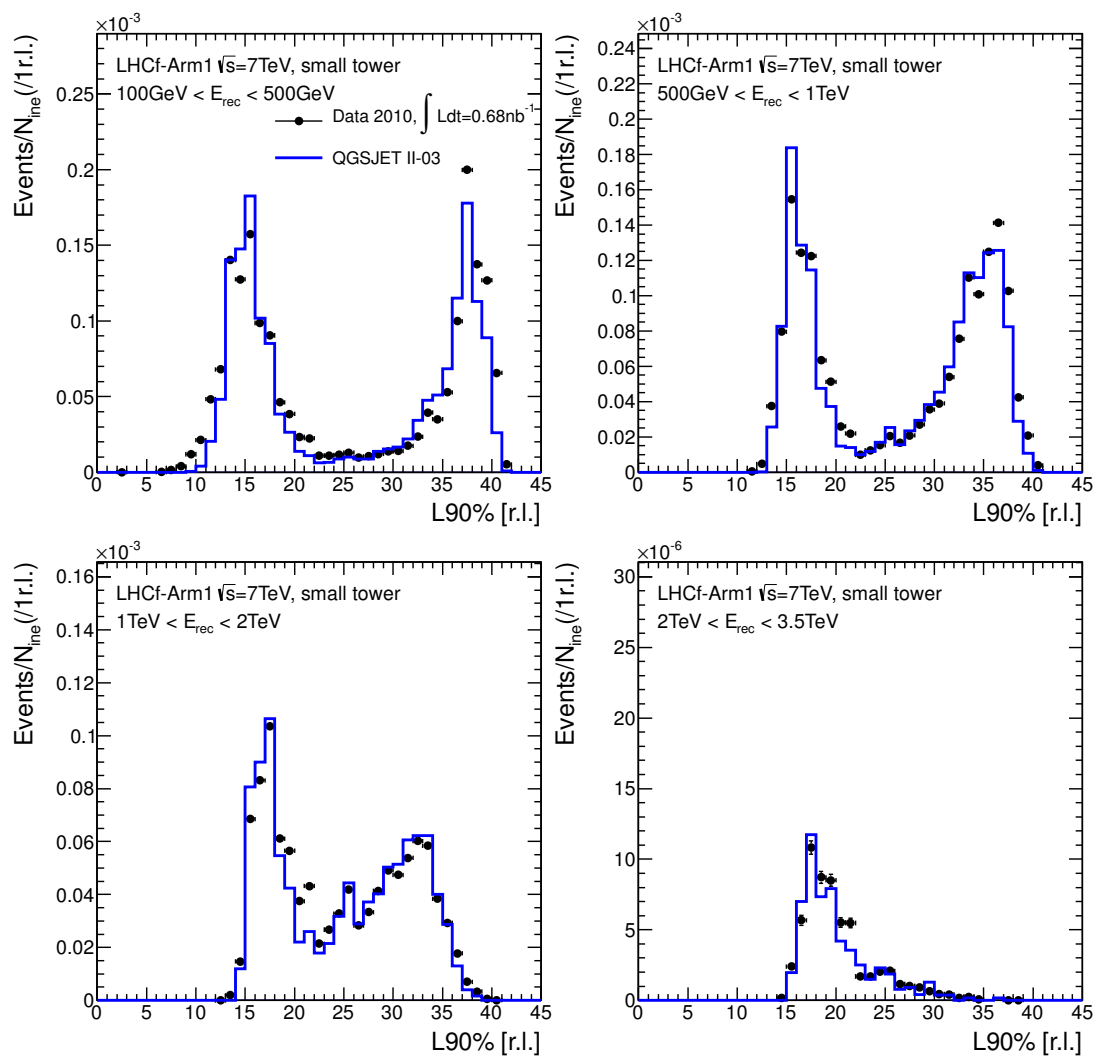

Fig. 12. $\mathrm{L}_{90 \%}$ distributions of the Arm1 small tower for different energy ranges. Data (crosses) and scaled templates (histograms) are shown.

The identification efficiency for the multi-hit photon-like events has been studied using the real data and MC simulations. Multi-hit events containing hadrons are identified as hadronic showers and eliminated from the photon event analysis in the PID process described in Sec.3.2 . Clear single photon-like events were selected from the data using the algorithm described above and two such events were superimposed based on the distributions of peak height ratio and lateral separation according to the DPMJET3 interaction model ${ }^{14}$. Applying the multi-hit identification algorithm with the criteria given above, the identification efficiency of the multi-hit (double-hit in this case) events is determined. Fig.16 shows the results for multi-hit identification efficiency as functions of the energy of the most energetic photon. Slightly energy dependent efficiencies of $>70 \%$ and $>90 \%$ are obtained for Arm1 and Arm2, respectively. The major sources of multi-hit events are combinatorial photons at lower energy and photon pairs produced in the $\pi^{0}$ decay above 1.5 TeV. Because the minimum angle between the photons from $\pi^{0}$ decay is kinematically defined, the separation of these photons are relatively easy and it results 

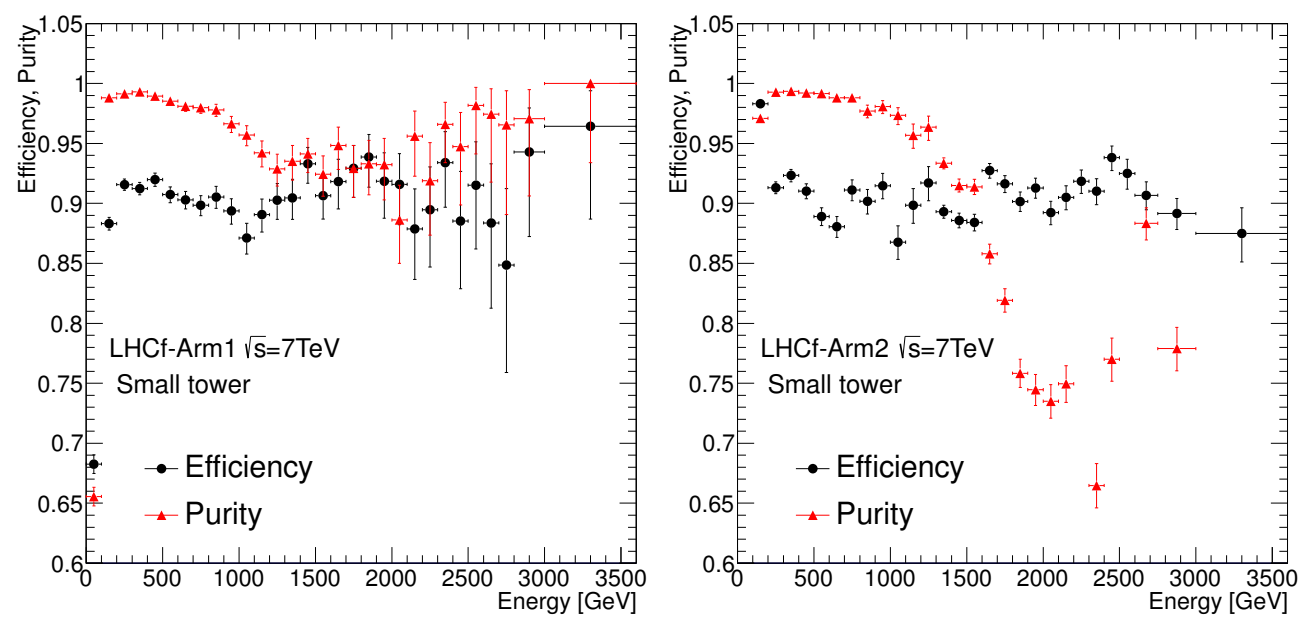

Fig. 13. Efficiency and purity of photon event selection in the $7 \mathrm{TeV}$ data analysis for the Arm1 (left) and Arm2 (right) small towers.
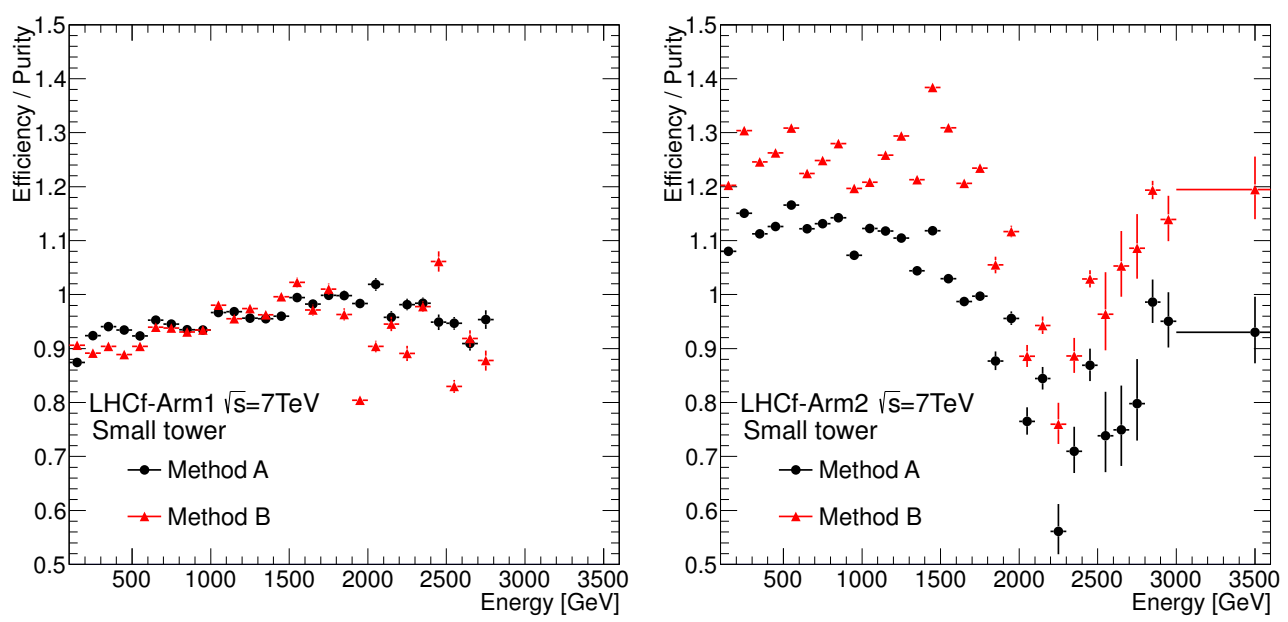

Fig. 14. PID correction factors estimated by two methods A and B. Left plots for Arm1 and right for Arm2.

higher efficiencies above $1.5 \mathrm{TeV}$. On the other hand, the combinatorial photons essentially hit the detector uniformly and have a certain probability of very close events those can be misidentified as a single-hit event. This results lower efficiencies below $1.5 \mathrm{TeV}$. Good agreement is found between the results based on the data and on the MC simulation using the EPOS interaction model ${ }^{15}$. 

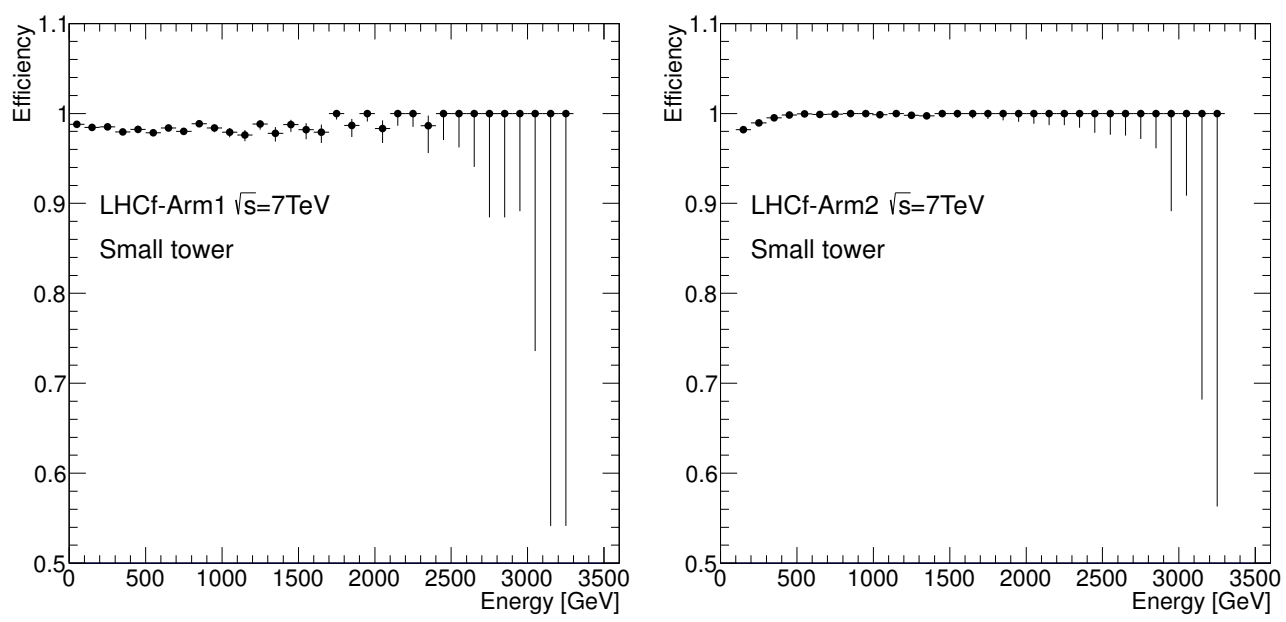

Fig. 15. Single hit selection efficiency for the $7 \mathrm{TeV}$ data analysis for Arm1 (left) and Arm2 (right)

\subsection{Beam Center}

In normal operation, the small towers were placed to cover the projection of zero degree emission angle relative to the directions of the colliding protons which we call 'beam center' hereafter. The ideal position of the beam center on the detectors was determined based on the survey after installation. However, the actual beam center can drift from time to time due to small differences in the beam trajectory causing an unintended beam crossing angle or transverse beam displacement. The real beam center can be measured by two methods. The first method uses the Beam Position Monitors (BPMs) installed $\pm 21.3 \mathrm{~m}$ from the interaction point ${ }^{16}$. The second method is to determine the center of the distribution of LHCf shower events. The determination of the real beam center is important in the LHCf analysis for correctly determining the rapidity and transverse momentum $\left(\mathrm{p}_{T}\right)$ of individual particle.

The data from the BPMs were recorded every 10 seconds through the Data Interchange Protocol (DIP). By using the beam positions in the horizontal and vertical directions at $\pm 21.3 \mathrm{~m}$ from the IP, the trajectories of the two beams passing through the IP can be determined. The beam centers at the LHCf detectors located $\pm 140 \mathrm{~m}$ away from the IP are determined by extrapolating the beam trajectories. Here we note that beam1 is directed toward Arm2 (on the IP2 side of IP1) and beam2 is directed toward Arm1 (on the IP8 side of IP1). The energy flux distributions of LHCf events were determined using the shower positions weighted by the primary particle energies measured event-by-event. Examples of energy flux distributions and fitting to the beam center are shown in Fig.17. The results of this quick analysis were published through the DIP during LHC operation. The beam center 

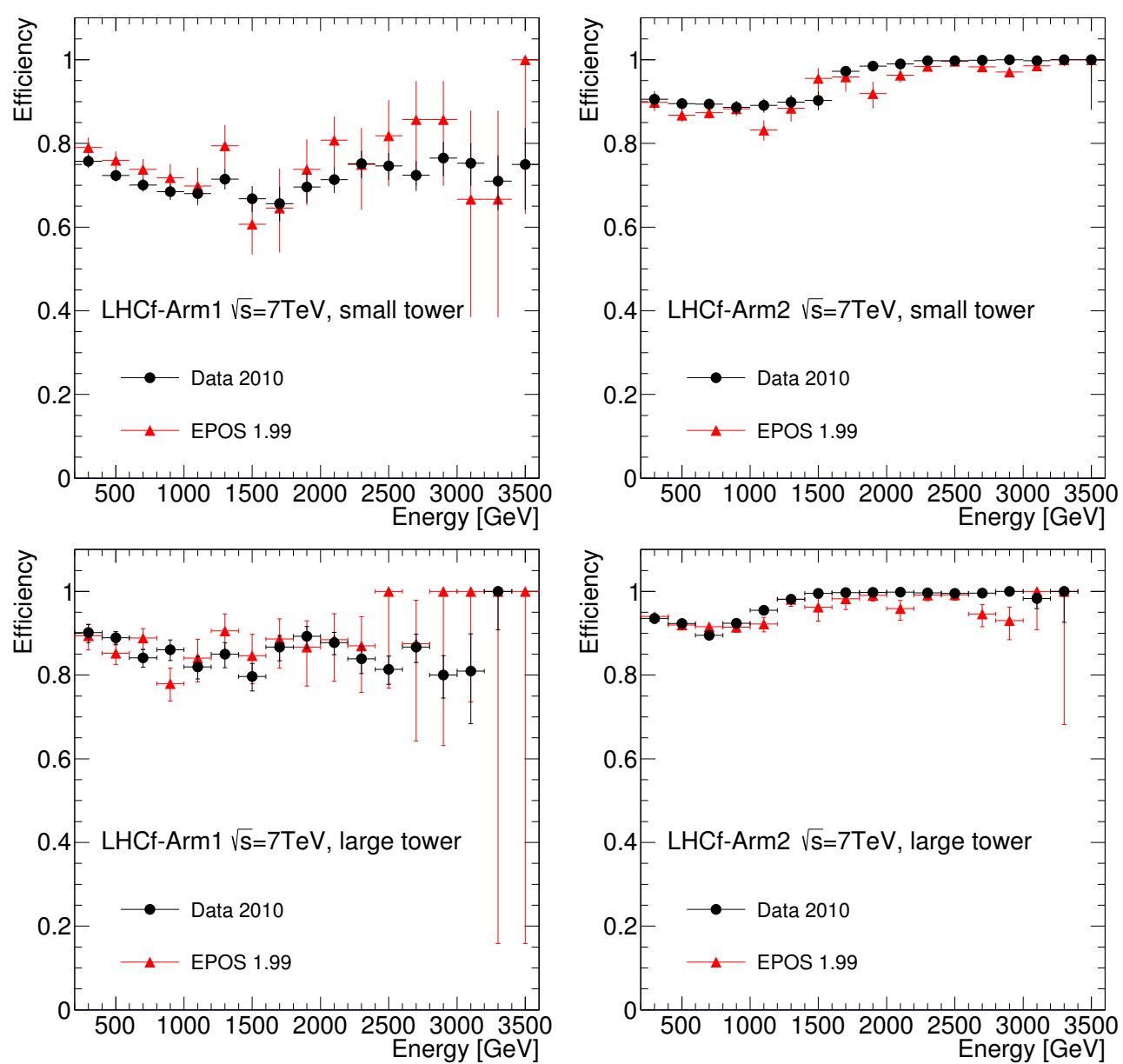

Fig. 16. Double-hit selection efficiency for the $7 \mathrm{TeV}$ data analysis for Arm1 (left) and Arm2 (right). Top and bottom plots show the results for the small and large towers, respectively. 'Data' designates the analysis results for the photon data superimposed according to the distributions of DPMJET3.

positions determined by BPMs and the LHCf detectors from fill 1002 to 1134 (from 30 Mar to 5 June, 2010) are plotted in Fig.18. Note that the beam energy for fills 1068, 1069 and 1128 was $450 \mathrm{GeV}$ while the beam energy was $3.5 \mathrm{TeV}$ for the remaining fills. Here the LHCf results with statistical uncertainty $<2 \mathrm{~mm}$ are plotted. Very good agreement of horizontal beam centers determined by BPM and LHCf data is found in Fig.18 for both the Arm1 and Arm2 sides. The typical difference in the two methods is smaller than $1 \mathrm{~mm}$.

On the other hand, some offsets between the BPM and LHCf methods in the vertical direction are found in Fig.18. Especially big offsets are found in the BPM results after fill 1080. The vertical beam position results determined by LHCf data, 
however, do not exhibit these offsets. If the $10 \mathrm{~mm}$ offset found in the BPM results of the Arm2 side were real, it should have easily been identified by the LHCf data according to the results shown in Fig.17. In the period before and after fill 1080 the beam intensity increased by a factor two. Because the calibrations of the BPMs in the early periods of LHC operation were known to have systematic uncertainties dependent on beam intensity, the large offsets of beam center in the vertical direction determined by the BPMs are not regarded as real after fill 1080 .

Once constant shifts of the BPM results before and after fill 1080 are accepted, very good agreements between the BPM and LHCf results for beam centers are found, again at the $1 \mathrm{~mm}$ level. Here $1 \mathrm{~mm}$ at the LHCf detector corresponds to a shift of beam position at the IP by $1 \mathrm{~mm}$ or of beam crossing angle by $7 \mu \mathrm{rad}$. A $1 \mathrm{~mm}$ uncertainty of the beam center position is used for estimating the systematic errors of the rapidity and $\mathrm{p}_{T}$ distributions determined by analysis of the LHCf data.

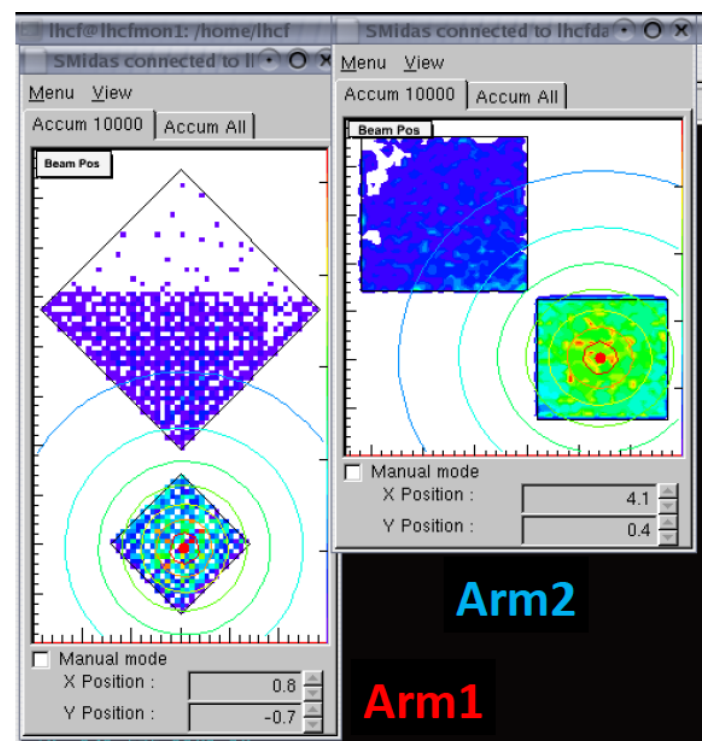

Fig. 17. The real time analysis results for the event position distribution and the fitting to the beam center during LHC operation. The definition of the coordinate system is same as Fig.9

\section{5. $\pi^{0}$ Identification and Energy Scale}

As shown in Fig.7, some events record a shower in each of the two towers. Assuming the two particles are photons and decay products from the IP, four momenta of the two photons and consequently the invariant mass of the pair, $m c^{2} \sim \theta \sqrt{E_{1} E_{2}}$, can be calculated. Here $\theta, \mathrm{E}_{1}$ and $\mathrm{E}_{2}$ are the opening angle and the energies of the two photons, respectively. In case of two photon events, some fraction of the shower particles leaks out from a tower and some of this leakage is detected in 

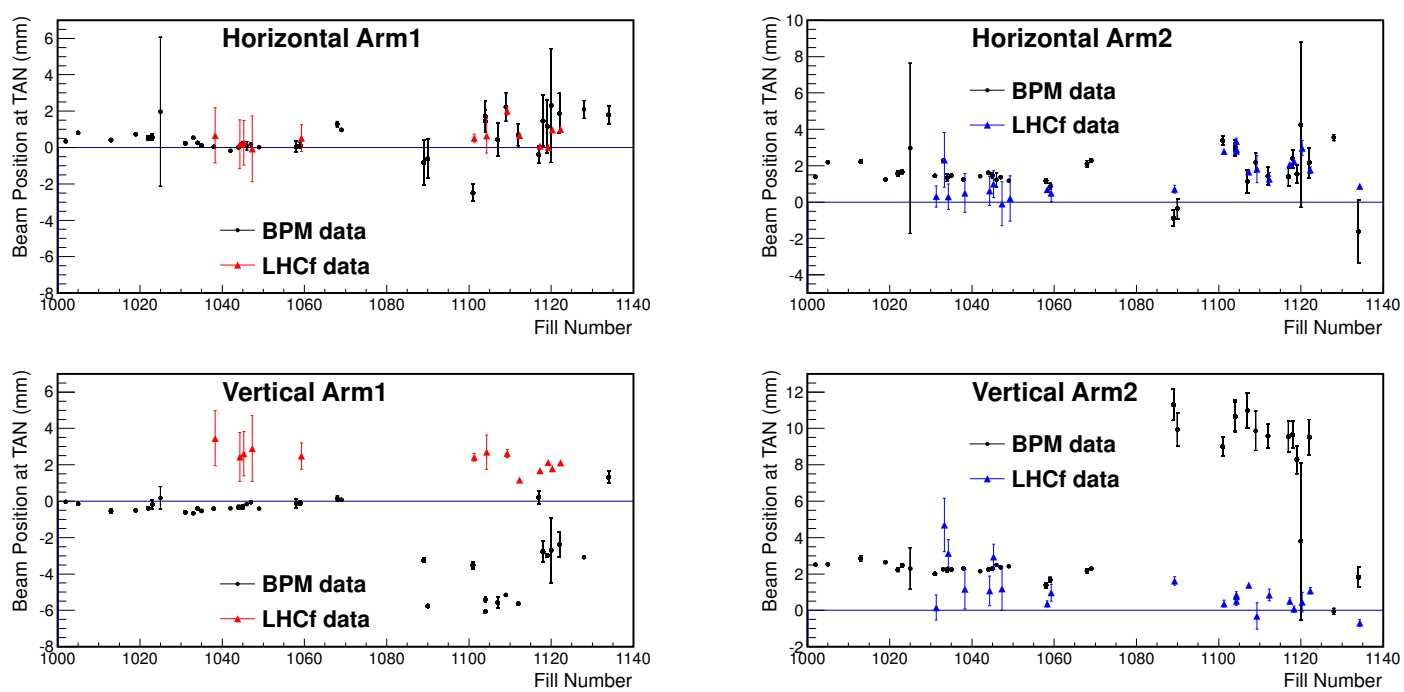

Fig. 18. Beam center at the position of the LHCf detectors determined from the LHCf data (triangles, red for Arm1 and blue for Arm2) and from the BPM data (black dots).

the other tower. This contamination called 'leak-in' is corrected using a correction factor determined by MC simulation and tested at SPS. Details of the $\pi^{0}$ analysis have been reported elsewhere ${ }^{10} 17$.

The invariant mass distribution reconstructed from the Arm2 data is shown in Fig.19. A clear peak above background near $140 \mathrm{MeV}$ corresponding to $\pi^{0}$ meson events and a marginal peak near $560 \mathrm{MeV}$ corresponding to $\eta$ meson events are found. Close-ups of the $\pi^{0}$ peaks for the Arm1 and Arm2 data are shown in Fig.20. Gaussian fitting ( $\sigma$ in the left and right sides of the peak are different in case of Arm1) to the peaks together with polynomial background fitting give the peak positions at $145.8 \pm 0.1 \mathrm{MeV}$ for Arm1 and $140.0 \pm 0.1 \mathrm{MeV}$ for Arm2 where the $\pm 0.1 \mathrm{MeV}$ errors are statistical. It should be noted that the known $\pi^{0}$ mass is $134.9766 \pm 0.0006 \mathrm{MeV}^{18}$ and the full MC simulation of the LHCf detectors and analysis gives peaks at $135.2 \pm 0.2 \mathrm{MeV}$ (Arm1) and $135.0 \pm 0.2 \mathrm{MeV}$ (Arm2). The 7.8\% (Arm1) and $3.7 \%$ (Arm2) mass excesses of the LHCf data exceed the statistical errors. An accumulation of the known systematic errors in the energy determination amounts to $\pm 3.5 \%$ and the uncertainty in shower position determination does not cause more than $1 \%$ error in the opening angle $\theta$ between the two photons. A leak-in correction owing to shower particles leaking out of one detector into the other has also been accounted for in the analysis. The systematic uncertainty in the leak-in correction has been estimated to be at the $2 \%$ level. The rms sum of these 'known' uncertainties, $\pm 4.2 \%$, can account for the mass peak excess in the Arm2 result within $1 \sigma$ but the mass peak excess in the Arm1 result amounts to $2 \sigma$.

The invariant mass peak positions as functions of the $\pi^{0}$ energy are plotted in 
Fig.21 for Arm1 and Arm2. The invariant mass excess is found to be independent of the $\pi^{0}$ energy in both detectors. This indicates the invent mass excess is not due to the extrapolation of the energy calibration from the energy scale of the SPS test beams to the energy scale of the LHC data but is a common energy error over the whole energy range.

The peak position corresponding to the $\eta$ meson is found at $562 \pm 2 \mathrm{MeV}, 2.6 \%$ higher than the known mass of $547.853 \pm 0.024 \mathrm{MeV}^{18}$. Because of the high $\eta$ mass, the opening angle of the decay photon pairs is larger than that of $\pi^{0}$. Due to the limited acceptance of the LHCf towers, the minimum energies of $\eta$ mesons detected in Arm1 and Arm2 during zero crossing angle operation are $2.9 \mathrm{TeV}$ and $2.2 \mathrm{TeV}$, respectively. The $\eta$ meson events are detected as photon pairs entering the detectors near the outer sides of the two towers. On the other hand, $\pi^{0}$ events with more than $600 \mathrm{GeV}$ are detected with a more uniform distribution of photon pairs across the faces of the towers. The hit position distributions of the photon pair for $\pi^{0}$ and $\eta$ candidate events are shown in Fig.22. So far the reason of the mass peak shifts has not been found and it is treated in the analysis as contribution to the systematic errors of the energy determination. Temperature dependence of the PMTs and scintillator responses that are larger than what we expected are possible sources of the mass peak shifts and are being studied.

\section{Background and Dose}

\subsection{Beam-gas background in the photon spectra}

Interactions between the beams and the residual gas in the vacuum beam pipe produce background events in the LHCf detectors. Because the LHCf detectors cannot identify the origin of each particle, the amount of beam-gas background is estimated using the events associated with the non-crossing bunches introduced in Sec.2.1. Until the removal of the LHCf detectors, the number of bunches stored in the LHC ring was between 2 and 13. Half of them were crossing bunches, one or two were non-crossing bunches and the others were crossing but longitudinally displaced bunches at IP1. The events associated with the displaced crossing bunches are not used in the current LHCf analysis. Identical analysis was applied to the events associated with both crossing and non-crossing bunches and the spectra are extracted separately. The photon spectra normalized with the beam intensity are shown in Fig.23. It is found that at maximum $10 \%$ and $<1 \%$ of beam-gas contamination exist in the $900 \mathrm{GeV}$ and $7 \mathrm{TeV}$ collision data, respectively.

\subsection{Dose in the towers}

During 2010 operation, the delivered luminosity at IP1 was estimated to be $350 \mathrm{nb}^{-1}$. According to a MARS calculation ${ }^{19}$ when the TAN slot is filled by copper bars instead of the LHCf detectors, the radiation dose is 180 MGy in 180 days of operation with a collision energy of $14 \mathrm{TeV}$ and a luminosity of $10^{34} \mathrm{~cm}^{-2} \mathrm{~s}^{-1}$. 


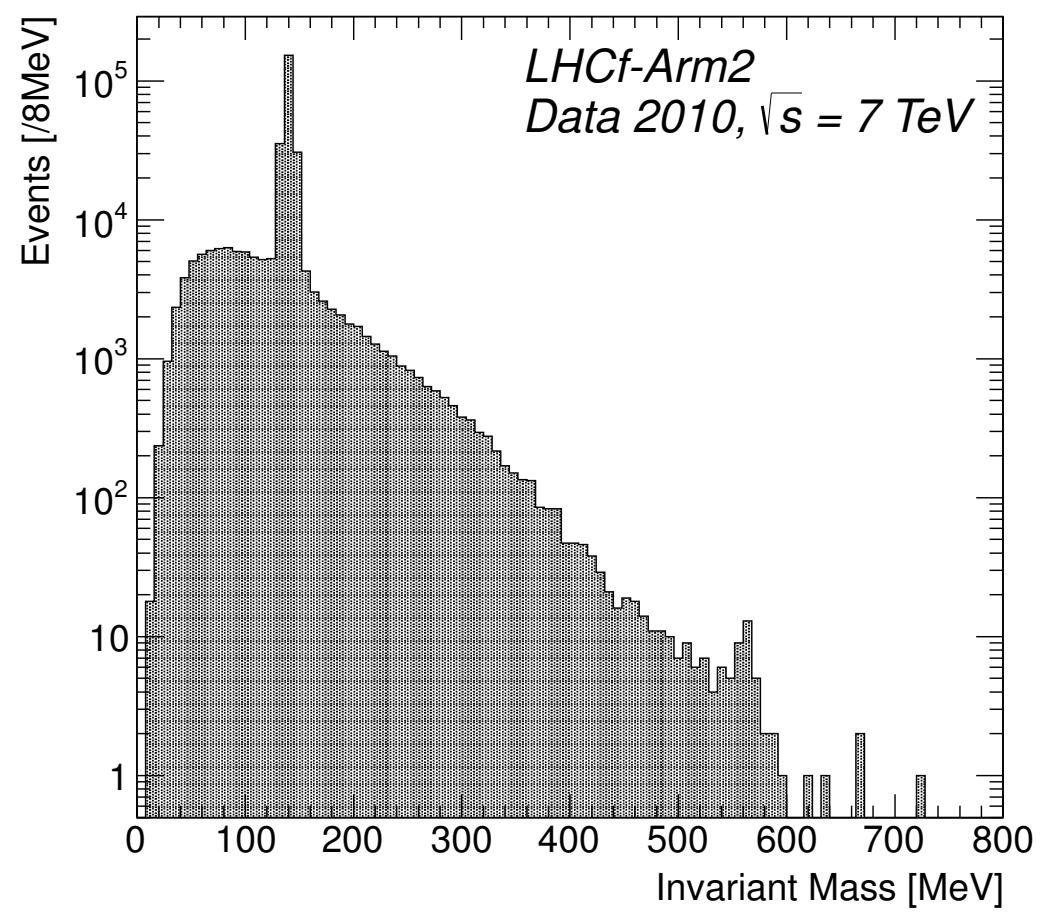

Fig. 19. Invariant mass distribution measured by the Arm2 detector.
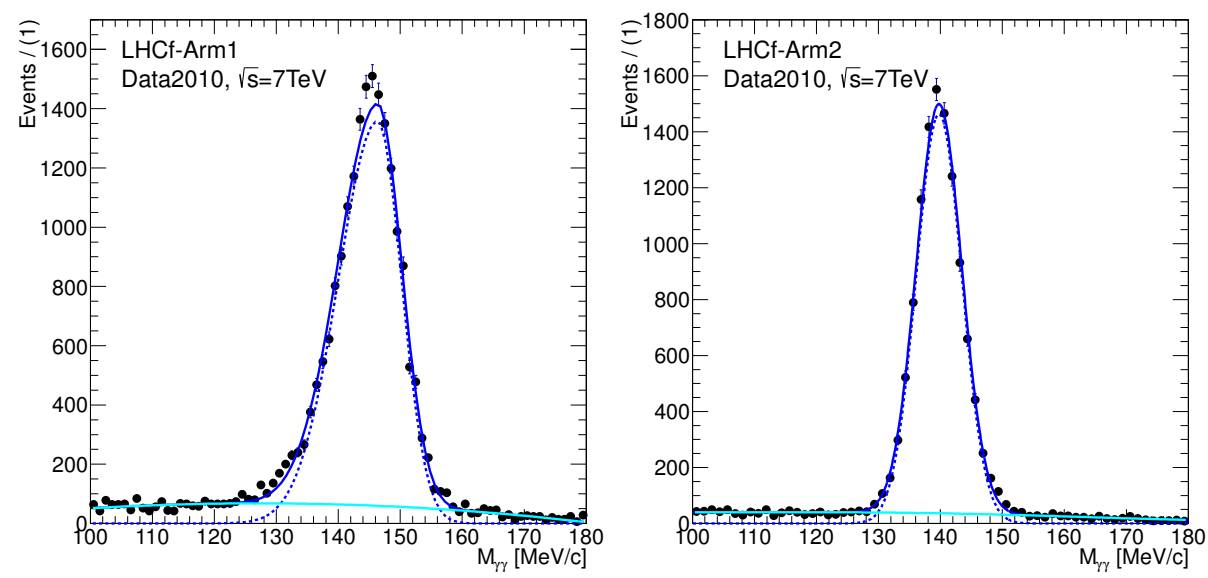

Fig. 20. Invariant mass distribution measured by the Arm1 (left) and Arm2 (right) detectors near the $\pi^{0}$ mass. 

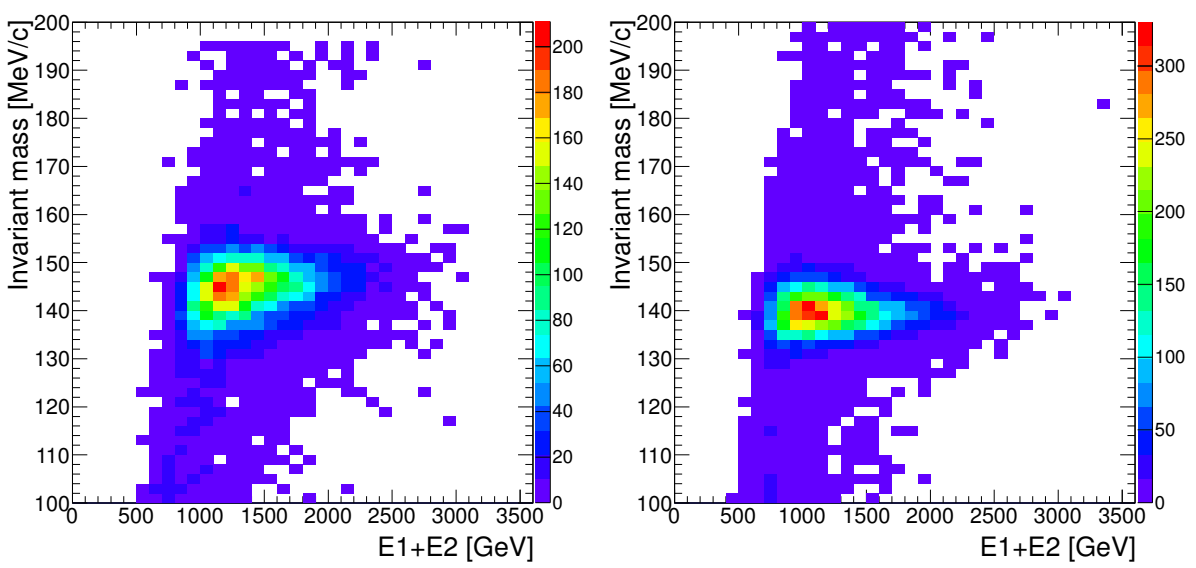

Fig. 21. Total photon energy and invariant mass distribution of the photon pair for Arm1 (left) and Arm2 (right).
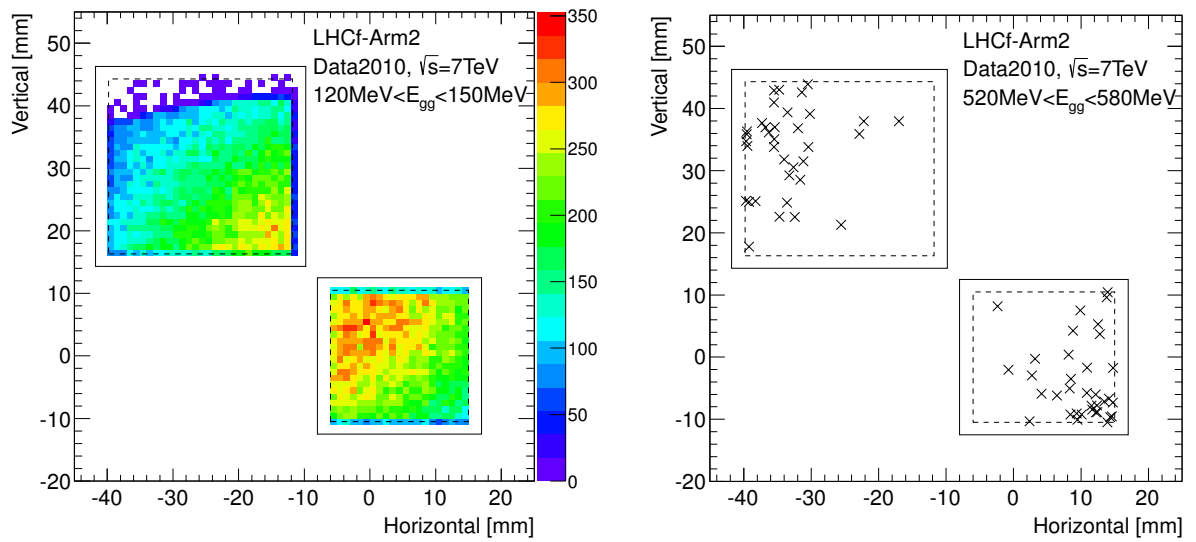

Fig. 22. Hit maps of the photon pairs having the invariant mass of the $\pi^{0}$ (left) and the $\eta$ (right). Color in the left figure shows number of photons and crosses in the right figure indicate individual photons.

This can be expressed as a dose of $1 \mathrm{~Gy} / \mathrm{nb}^{-1}$ at a depth of 12 radiation lengths in copper.

LHCf has estimated the radiation dose during $7 \mathrm{TeV}$ collisions by using the total measured energy deposited in the scintillators. Minimum biased data taken with $\mathrm{L}_{2} \mathrm{~T}_{L 1 T}$ trigger (trigger by beam passage but without requiring interaction) are analyzed. The average dose rate in a $20 \mathrm{~mm} \times 20 \mathrm{~mm}$ scintillator covering zero degree collision angle and placed behind an absorber of 1 radiation length of copper plus 10 radiation lengths of tungsten was $0.2 \mathrm{~Gy} / \mathrm{nb}^{-1}$ after correcting for the dead 

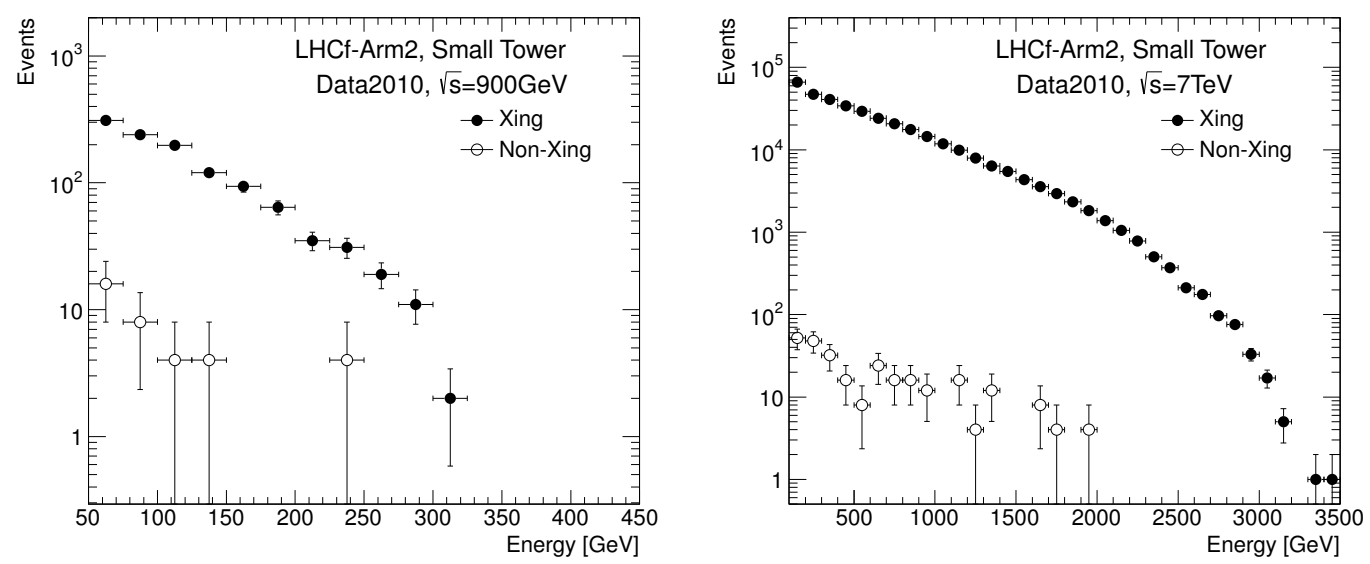

Fig. 23. Energy spectra of photons associated with the crossing bunches (filled circles) and noncrossing bunches (open circles) normalized with the beam intensity.

time of the data acquisition system. After $350 \mathrm{nb}^{-1}$ of operation a radiation dose of $70 \mathrm{~Gy}$ is expected. The effect of radiation damage is discussed in Sec.5.

Another measurement of radiation dose was made by dosimeters placed behind the small towers. The time-histories of dosimeters during 2010 operation are shown in Fig.24. Dosimeters provide continuous dose measurements that are independent of the trigger setup. According to Fig.?? the accumulated radiation dose measured by dosimeters placed behind the small towers was 6 and 10 Gy for Arm1 and Arm2, respectively.

\section{Data Stability}

According to a previous study ${ }^{4}$, a $10 \%$ decrease in signal is expected for EJ-260 plastic scintillator to $100 \mathrm{~Gy}$ of irradiation. In the actual situation of LHCf the irradiation was not continuous over the three months of operation and the scintillators exhibit recovery from damage during non-irradiation periods. The stability of the plastic scintillator response during 2010 operation was studied by monitoring the reconstructed invariant mass peak corresponding to $\pi^{0}$ mesons and by irradiating the scintillators with a UV laser. The time history of the reconstructed mass peak of $\pi^{0}$ events is plotted in Fig.25. An overall $3 \%$ decrease in the peak position is found in both the Arm1 and Arm2 results from the beginning of irradiation to the end in Fig.25, although the decrease is not monotonic owing to recovery during non-irradiation periods. This magnitude of the decrease is in reasonable agreement with expectations from the radiation damage. The scintillators and PMTs have also been observed to have temperature dependences that could effect signal stability and need further study before quantitative statements can be made.

The edges of the scintillators were irradiated with a $337 \mathrm{~nm}$ pulsed laser during 


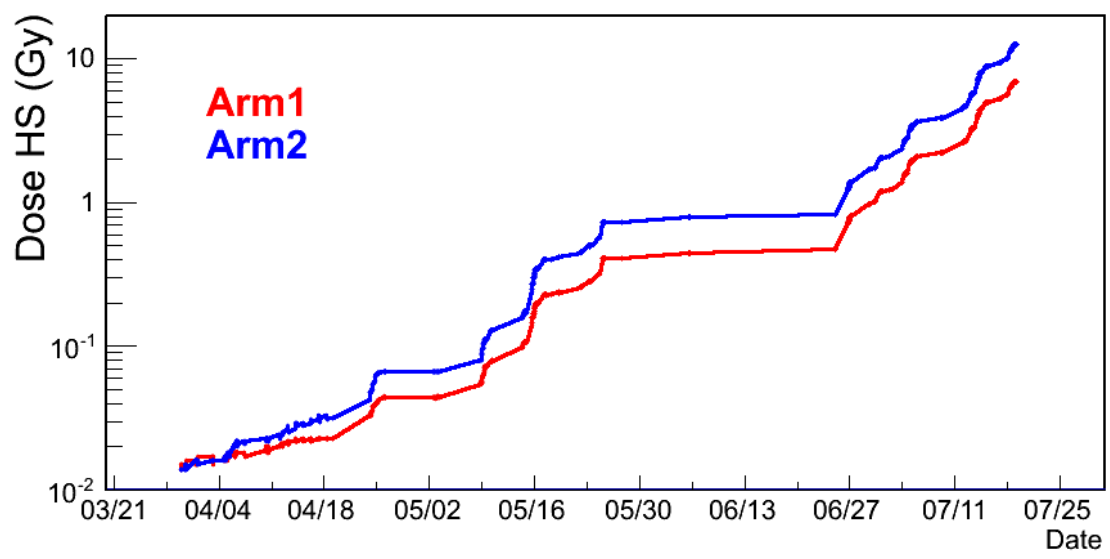

Fig. 24. Accumulated radiation doses measured behind the small towers. HS designates the results of the high sensitivity dosimeters.

beam off periods. The results of long term monitoring using UV light for exciting the scintillators at a depth of 8 radiation lengths during 2010 operation are shown in Fig.26. While a trend of decreasing signal with increasing irradiation is seen in the Arm2 results of Fig.26, this trend does not appear in the Arm1 results. Because the accuracy of the laser monitoring is about $\pm 5 \%$ as indicated by dashed lines, we conclude there was no large change in the scintillator and PMT responses during 2010 operation.

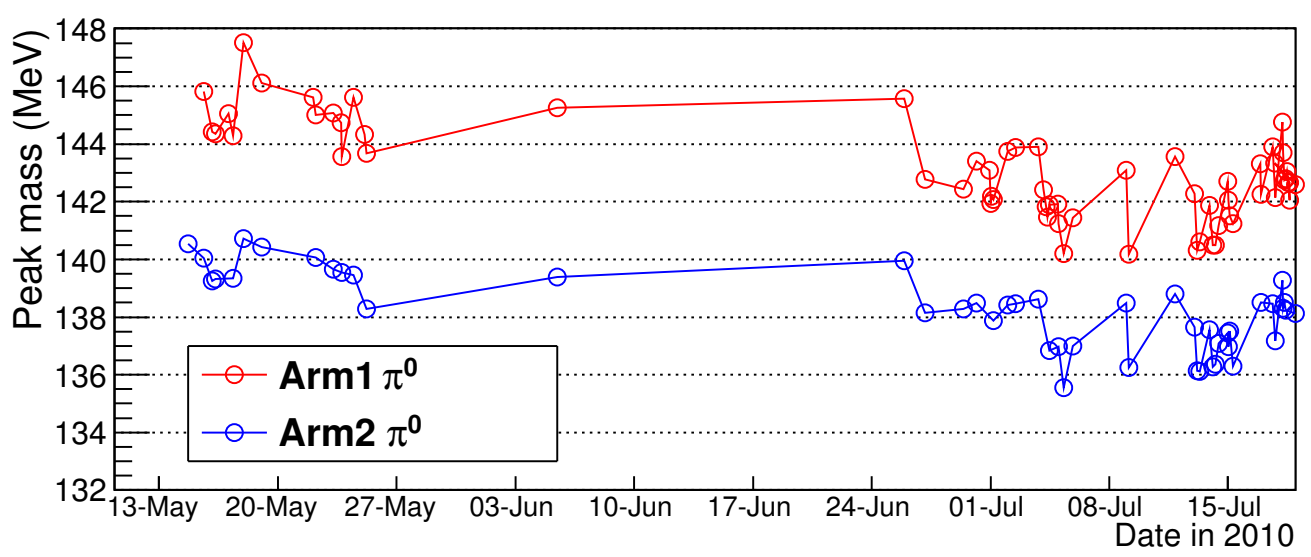

Fig. 25. The time histories of the reconstructed $\pi^{0}$ mass. 

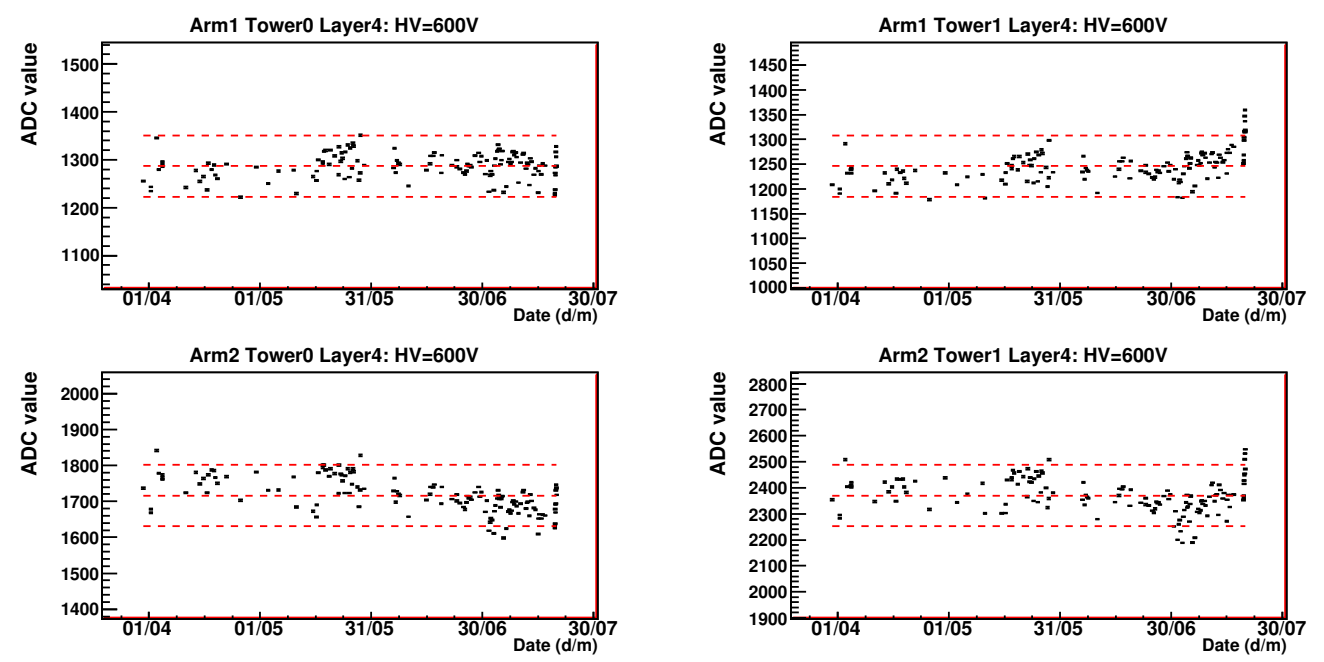

Fig. 26. Long term stabilities of the four scintillator layers and PMTs monitored by a UV laser. Three horizontal lines indicate the average and $\pm 5 \%$ deviation from the average which is the accuracy of the method.

\section{Summary}

LHCf successfully finished its first period of operation at LHC for $900 \mathrm{GeV}$ and $7 \mathrm{TeV}$ p-p collisions in 2009 and 2010. During the 3.5 months of operation in 2010 LHCf collected collision data of $900 \mathrm{GeV}$ and $7 \mathrm{TeV}$ collisions corresponding to delivered luminosities of $0.3 \mathrm{nb}^{-1}$ and $350 \mathrm{nb}^{-1}$, respectively. Following this operation the LHCf detectors were removed from the LHC tunnel to protect them from radiation damage with the increasing luminosity of LHC. The overall detector performance for determining the luminosity, the incident energy and position, the particle type, the single hit and multi-hit events and the beam center satisfies the requirements for obtaining LHCf physics results for photon and neutral pion production spectra near zero degrees. Reconstruction of the invariant mass of $\pi^{0}$ events showed unexpected upward mass shifts compared to the known $\pi^{0}$ mass. The reason for these shifts is not yet understood and a systematic error in the published spectra is included to account for these shifts. The contamination of collision data with beam-gas background was studied by using data with non-crossing bunches. It is found that the beam-gas contamination of the final photon spectra is at maximum $10 \%$ in the $900 \mathrm{GeV}$ spectra and $<1 \%$ in the $7 \mathrm{TeV}$ spectra. The long term stability of the detector response was also studied by monitoring the reconstructed $\pi^{0}$ mass peak and the response to laser irradiation. A gradual decrease of $3 \%$ in the signal strength, consistent with the expectation from radiation damage, was observed by monitoring the $\pi^{0}$ mass peak. Laser monitoring also assured no abrupt change of signal strength during 2010 operation although the accuracy was limited to $\pm 5 \%$.

Overall 2009-2010 operation satisfied the requirements for obtaining LHCf 
physics results $89^{10}$. Further understanding of the detector performance, in particular the unknown cause of upward shifts of the $\pi^{0}$ mass, would reduce the systematic uncertainty of the already published results of single photon and $\pi^{0}$ production spectra near zero degree collision angle.

\section{Acknowledgments}

We thank the CERN staff and the ATLAS collaboration for their essential contributions to the successful operation of LHCf. This work was partly supported by Grant-in-Aid for Scientific Research by MEXT of Japan, the Mitsubishi Foundation in Japan and INFN in Italy. The receipts of a JSPS Research Fellowship (HM), INFN fellowships for non-Italian citizens (HM and KN) and the GCOE Program of Nagoya University 'QFPU' from JSPS and MEXT of Japan (GM) are also acknowledged. A part of this work was performed using the computer resources provided by the Institute for the Cosmic-Ray Research (ICRR), University of Tokyo.

\section{References}

1. LHCf Technical Design Report, CERN-LHCC-2006-004.

2. M. Mizuishi et al., J. Phys. Soc. Jpn 78, Suppl. A, 173 (2009).

3. O. Adriani et al., JINST 5, P01012 (2010).

4. O. Adriani et al., JINST 3, S08006 (2008).

5. T. Sako et al., NIM A578, 146 (2007).

6. T. Mase et al., NIM A671,129 (2012).

7. K. Taki et al., JINST 7, T01003 (2012).

8. O.Adriani et al., Phys. Lett. B 703, 128 (2011).

9. O. Adriani et al., Phys. Lett. B 715, 298 (2012).

10. O. Adriani et al., Phys. Rev. D, in press (2012).

11. M. Morhac et al., NIM A401, 113 (1997); M. Morhac et al., NIM A401, 385 (1997); M. Morhac et al., NIM A443, 108 (2000).

12. R. Brun and F. Rademakers, ROOT - An Object Oriented Data Analysis Framework, Proceedings AIHENP' 96 Workshop, Lausanne, Sep. 1996, NIM 389, 81 (1997). See also http://root.cern.ch/.

13. S. Ostapchenko, Phys. Rev. D 74, 014026 (2006).

14. F.W. Bopp, J. Ranft, R. Engel, S. Roesler, Phys. Rev. C 77, 014904 (2008).

15. K. Werner, F.-M. Liu, T. Pierog, Phys. Rev. C 74, 044902 (2006).

16. G. Vismara, CERN-SL-2000-056 BI, 2000.

17. Menjo et al., Astropart. Phys. 34, 513 (2011)

18. Particle Data Group, J. Phys. G 37, 075021 (2010).

19. N. Mokhov, LHC Project Report 633. 